\title{
OPTIMIZED SCHWARZ COUPLING OF BIDOMAIN AND MONODOMAIN MODELS IN ELECTROCARDIOLOGY
}

\author{
Luca Gerardo-Giorda $^{1}$, Mauro Perego ${ }^{1}$ and Alessandro Veneziani ${ }^{1}$
}

\begin{abstract}
The Bidomain model is nowadays one of the most accurate mathematical descriptions of the action potential propagation in the heart. However, its numerical approximation is in general fairly expensive as a consequence of the mathematical features of this system. For this reason, a simplification of this model, called Monodomain problem is quite often adopted in order to reduce computational costs. Reliability of this model is however questionable, in particular in the presence of applied currents and in the regions where the upstroke or the late recovery of the action potential is occurring. In this paper we investigate a domain decomposition approach for this problem, where the entire computational domain is suitably split and the two models are solved in different subdomains. Since the mathematical features of the two problems are rather different, the heterogeneous coupling is non trivial. Here we investigate appropriate interface matching conditions for the coupling on non overlapping domains. Moreover, we pursue an Optimized Schwarz approach for the numerical solution of the heterogeneous problem. Convergence of the iterative method is analyzed by means of a Fourier analysis. We investigate the parameters to be selected in the matching radiation-type conditions to accelerate the convergence. Numerical results both in two and three dimensions illustrate the effectiveness of the coupling strategy.
\end{abstract}

Mathematics Subject Classification. 65M55, 65N30, 92-08.

Received September 5, 2009. Revised February 27, 2010.

Published online August 20, 2010.

\section{INTRODUCTION}

The Bidomain model is nowadays one of the most accurate mathematical descriptions of the action potential propagation in the heart. In the original formulation this system features two nonlinear reaction-diffusion parabolic equations for the extra and intra-cellular potentials. The matrix multiplying the time derivatives of the potentials is singular and for this reason the system is called "degenerate". Mathematical features of this model induce some difficulties in its numerical approximation that lead to ill conditioned problems. For this reasons many efforts have been devoted to the set up of efficient solvers and preconditioners $[4,7,27,39,40]$, possibly based on parallel multigrid approaches [26,33,41] or suitable approximations of the equations [16]. Another approach has been aiming at a simplification of the original problem. In particular, under a simplifying assumption on the anisotropy in the intra- and extracellular spaces, the two equations of the Bidomain system can be combined

\footnotetext{
Keywords and phrases. Computational electrocardiology, Optimized Schwarz Methods, heterogeneous models.

1 Dept. of Mathematics and Computer Science, Emory University, Atlanta, USA. luca@mathcs.emory.edu;

mauro@mathcs.emory.edu; ale@mathcs.emory.edu
} 
yielding a single nonlinear reaction-diffusion parabolic equation in the transmembrane potential. This is called Monodomain problem and it is clearly simpler to solve. However, it has been pointed out that this model fails in capturing some remarkable features of the action potential propagation [37].

In this paper we investigate the coupling of the two problems, the Bidomain and Monodomain ones solved in different parts of the computational domain. The basic idea behind this heterogeneous approach is to reduce computational costs and to maintain the accuracy by solving the Bidomain problem over "critical" (and hopefully small) regions of the domain (where the term "critical" is driven by physiopathological arguments). The Monodomain equation is solved in parts where the potential propagation dynamics does not require the most sophisticated model. This approach falls in the general framework of "model adaptivity". In this direction, a sort of intermediate model called Hybridomain has been recently proposed in [23]. This model relies on an extended formulation of the Monodomain problem (formerly used in [16] as preconditioner for the Bidomain one). Should an appropriate error estimator be available, model adaptivity with Hybridomain is pretty immediate. Unfortunately, computational costs reduction is partially limited by the adoption of the extended Monodomain equations. We work here in the direction of a model adaptive strategy, in which the solver is able to adaptively select at each time step the regions where the Bidomain problem has to be solved, and the ones where the simplified Monodomain model is sufficiently accurate. This requires to solve the heterogeneous Bidomain/Monodomain problem. We face this issue with a Domain Decomposition (DD) approach. Coupling of the two problems is non trivial for their different nature. Here, we address a possible set of matching conditions. The basic idea is to start from a homogeneous Bidomain-Bidomain DD problem and then to downscale the Bidomain to the Monodomain model on one of the subdomains. The downscaling process is performed also on the matching conditions originally devised for the homogeneous problem.

We cope with the numerical solution of the heterogeneous problem by means of an Optimized Schwarz Algorithm. This approach is based upon Robin-type transmission conditions on the interface between subdomains. Differently from the classical Schwarz Algorithm, based on Dirichlet transmission conditions, Optimized Schwarz Algorithms converge also on nonoverlapping subdomains, and show a significant improvement in terms of both robustness and computational cost with respect to the classical Schwarz one (see for instance $[11,12,14,15]$ ). We perform a Fourier analysis of the problem in order to find the optimal parameters to be used in the Robintype interface conditions, i.e. the parameters that maximize the convergence rate of the iterative DD scheme. Several numerical results are presented both in $2 \mathrm{D}$ and 3D, including the simulation of a stimulus exerted by a pacemaker on a simplified left ventricular geometry. Results confirm that the coupled Bidomain/Monodomain problem is a viable approach for reducing computational costs and maintaining good accuracy, provided that the Bidomain model is activated in "critical" regions, e.g. the regions where the upstroke or the late recovery of the action potential are occurring, or in the presence of applied currents.

The outline of the paper is as follows. In Section 2 we briefly recall the Bidomain and Monodomain models for the description of action potential propagation in myocardial cells and the discretization methods we adopted (for more details see e.g. $[7,31,40])$. In Section 3 we consider the "Extended Monodomain" model as an intermediate model for devising the coupling conditions on the interface between the Bidomain region and the Monodomain region. In Section 4 we describe the Optimized Schwarz Method, we analyze its convergence via Fourier analysis and we address its algebraic formulation. Finally, Section 5 presents numerical results in both two and three dimensions that show the effectiveness of the method.

\section{A SURVEY OF MATHEMATICAL AND NUMERICAL MODELS IN ELECTROCARDIOLOGY}

\subsection{Description of the problem}

The myocardial tissue is composed of elongated cells, connected each other in fibers by gap junctions and surrounded by an extracellular medium. The conductivity of the cardiac cells depends upon their orientation, and in the most general case the conductivity tensor is anisotropic (see $e . g$. [20,35]). In any point $\boldsymbol{x} \in \Omega$, where $\Omega$ is the spatial domain under consideration, it is thus possible to identify an orthonormal triplet of directions, 
$\boldsymbol{a}_{l}(\boldsymbol{x}), \boldsymbol{a}_{t}(\boldsymbol{x}), \boldsymbol{a}_{n}(\boldsymbol{x})$, with $\boldsymbol{a}_{l}(\boldsymbol{x})$ parallel to the fibers direction, $\boldsymbol{a}_{t}(\boldsymbol{x})$ and $\boldsymbol{a}_{n}(\boldsymbol{x})$ tangent and orthogonal respectively to the radial lamination, both transversal with respect to the fiber axis.

\subsubsection{The Bidomain model}

In the Bidomain model the cardiac tissue is regarded as the superposition of two continuous and anisotropic media, the intra-cellular and the extra-cellular one. They do coexist at each point $\boldsymbol{x}$ and are connected by a cell membrane dislocated in the domain (for more details see $[8,18]$ ). The intra and extracellular media feature different conductivity values in each direction. Denoting by $\sigma_{\tau}^{l}, \sigma_{\tau}^{t}$, and $\sigma_{\tau}^{n}(\tau=i, e)$, the intra and extracellular conductivity coefficients respectively, in the $\boldsymbol{a}_{l}, \boldsymbol{a}_{t}$ and $\boldsymbol{a}_{n}$ directions, the conductivity tensors are given by

$$
\mathbf{D}_{\tau}(\boldsymbol{x})=\sigma_{\tau}^{l}(\boldsymbol{x}) \boldsymbol{a}_{l}(\boldsymbol{x}) \boldsymbol{a}_{l}^{T}(\boldsymbol{x})+\sigma_{\tau}^{t}(\boldsymbol{x}) \boldsymbol{a}_{t}(\boldsymbol{x}) \boldsymbol{a}_{t}^{T}(\boldsymbol{x})+\sigma_{\tau}^{n}(\boldsymbol{x}) \boldsymbol{a}_{n}(\boldsymbol{x}) \boldsymbol{a}_{n}^{T}(\boldsymbol{x}), \quad \tau=i, e .
$$

Under the assumption of axial isotropy, i.e. the direction across fibers features the same conductivity in both the tangential and normal direction, the tensors simplify in

$$
\mathbf{D}_{\tau}(\boldsymbol{x})=\sigma_{\tau}^{t} \mathbf{I}+\left(\sigma_{\tau}^{l}-\sigma_{\tau}^{t}\right) \boldsymbol{a}_{l}(\boldsymbol{x}) \boldsymbol{a}_{l}^{T}(\boldsymbol{x})
$$

We assume (2.2) to hold (see [7]). Moreover, we assume that $\mathbf{D}_{\tau}$ fulfills in $\Omega$ a uniform elliptic condition.

Let $u_{i}$ and $u_{e}$ be the intra and extracellular potentials respectively and $u=u_{i}-u_{e}$ be the transmembrane potential. The density current in each domain can be computed as $\mathbf{J}_{\tau}=-\mathbf{D}_{\tau} \nabla u_{\tau}, \tau=i, e$. The net current flux between the intra and the extracellular domain is assumed to be zero as a consequence of the charge conservation in an arbitrary portion of tissue. Let us denote by $I_{m}$ the ingoing membrane current flow and by $\chi$ the ratio of membrane area per tissue volume. We obtain

$$
\nabla \cdot\left(\mathbf{D}_{i} \nabla u_{i}\right)=\chi I_{m}=-\nabla \cdot\left(\mathbf{D}_{e} \nabla u_{e}\right)
$$

where $I_{m}=C_{m} \mathrm{~d} u / \mathrm{d} t+I_{\text {ion }}(u, \boldsymbol{w})$ being $C_{m}$ a capacitance and $I_{\text {ion }}$ the ionic current, depending on the potential $u$ and on suitable ionic variables that we denote with $\boldsymbol{w}$. The complete Bidomain model reads

$$
\left\{\begin{array}{l}
\chi C_{m} \frac{\partial u}{\partial t}-\nabla \cdot \mathbf{D}_{i} \nabla u_{i}+\chi I_{\text {ion }}(u, \boldsymbol{w})=I_{i}^{\text {app }} \\
-\chi C_{m} \frac{\partial u}{\partial t}-\nabla \cdot \mathbf{D}_{e} \nabla u_{e}-\chi I_{\text {ion }}(u, \boldsymbol{w})=-I_{e}^{\text {app }}
\end{array}\right.
$$

where $I_{\text {ion }}(u, \boldsymbol{w})$ is a nonlinear function of the transmembrane potential $u$, specified by a ionic model (in our numerical tests we consider the Luo-Rudy Phase I model for ventricular cells [22]), and where $I_{i, e}^{\text {app }}$ are applied external stimuli. The problem is completed by initial conditions, $u_{i}(\boldsymbol{x}, 0)=u_{i, 0}, u_{e}(\boldsymbol{x}, 0)=u_{e, 0}, u(\boldsymbol{x}, 0)=$ $u_{i, 0}-u_{e, 0}$ and by homogeneous Neumann boundary conditions on $\partial \Omega$, modelling an insulated myocardium,

$$
\mathbf{n}^{T} \mathbf{D}_{i} \nabla u_{i}(\boldsymbol{x}, t)=0 \quad \text { and } \quad \mathbf{n}^{T} \mathbf{D}_{e} \nabla u_{e}(\boldsymbol{x}, t)=0, \quad \text { on } \partial \Omega \times(0, T),
$$

where $\mathbf{n}$ is the unit normal outward-pointing vector on the surface. As a consequence of the Gauss theorem, the applied external stimuli must fulfill the compatibility condition

$$
\int_{\Omega} I_{i}^{\mathrm{app}} \mathrm{d} \boldsymbol{x}=\int_{\Omega} I_{e}^{\mathrm{app}} \mathrm{d} \boldsymbol{x} .
$$

System (2.4) consists of two parabolic reaction diffusion equations for $u_{i}$ and $u_{e}$ where the vector of time derivatives is multiplied by a singular matrix. The system is thus said to be degenerate. The transmembrane potential $u$ is uniquely determined, while the intra and extracellular potentials $u_{i}$ and $u_{e}$ are determined up 
to the same function of time, whose value is usually obtained by imposing that $u_{e}$ has zero mean on $\Omega$. For well-posedness analysis of the Bidomain problem associated with different ionic models see $[2,3,8,38]$.

In what follows we will rely on a non-symmetric formulation (2.8) (see e.g. [16]). Let us define

$$
\lambda_{m}=\min \left\{\frac{\sigma_{e}^{l}}{\sigma_{i}^{l}}, \frac{\sigma_{e}^{t}}{\sigma_{i}^{t}}\right\} \quad \lambda_{M}=\max \left\{\frac{\sigma_{e}^{l}}{\sigma_{i}^{l}}, \frac{\sigma_{e}^{t}}{\sigma_{i}^{t}}\right\}
$$

and we let $\lambda_{m} \leq \lambda \leq \lambda_{M}$. By linear combinations of the two equations in (2.4), with coefficients $\left(\frac{\lambda}{1+\lambda},-\frac{1}{1+\lambda}\right)$, and $(1,1)$, Bidomain system can be reformulated in non-symmetric form, in terms of the transmembrane and the extracellular potentials $u$ and $u_{e}$,

$$
\left\{\begin{array}{l}
\chi C_{m} \frac{\partial u}{\partial t}-\nabla \cdot\left(\frac{\lambda \mathbf{D}_{i}}{1+\lambda} \nabla u\right)-\nabla \cdot\left(\frac{\lambda \mathbf{D}_{i}-\mathbf{D}_{e}}{1+\lambda} \nabla u_{e}\right)+\chi I_{\text {ion }}(u, \boldsymbol{w})=I^{\text {app }} \\
-\nabla \cdot\left[\mathbf{D}_{i} \nabla u+\left(\mathbf{D}_{i}+\mathbf{D}_{e}\right) \nabla u_{e}\right]=\widetilde{I}^{\text {app }}
\end{array}\right.
$$

where we have set $I^{\text {app }}=\frac{\lambda I_{i}^{\text {app }}+I_{e}^{\text {app }}}{1+\lambda}$ and $\widetilde{I}^{\text {app }}=I_{i}^{\text {app }}-I_{e}^{\text {app }}$.

\subsubsection{The Monodomain model}

Derivation of the Monodomain model can be obtained in different ways, based upon a proportionality assumption between the intracellular and the extracellular conductivity tensors, namely assuming $\mathbf{D}_{e}=\lambda \mathbf{D}_{i}$, where $\lambda$ is a constant to be properly chosen. For instance, under assumption (2.2), $\lambda$ can be devised through a minimization procedure, as

$$
\lambda=\operatorname{argmin} J(\lambda), \quad J(\lambda)=\left(\frac{\sigma_{e}^{l}-\lambda \sigma_{i}^{l}}{1+\lambda}\right)^{2}+2\left(\frac{\sigma_{e}^{t}-\lambda \sigma_{i}^{t}}{1+\lambda}\right)^{2}
$$

for given values of the conductivities. A time dependent choice of the parameter $\lambda$ has been proposed in [24].

After defining $\mathbf{D}:=\mathbf{D}_{i}+\mathbf{D}_{e}$ and $\mathbf{D}_{M}:=\mathbf{D}_{e} \mathbf{D}^{-1} \mathbf{D}_{i}$, the first equation in (2.8) can be rearranged as

$$
\chi C_{m} \frac{\partial u}{\partial t}-\nabla \cdot \mathbf{D}_{M} \nabla u+\nabla \cdot\left[\left(\mathbf{D}_{e} \mathbf{D}^{-1}-\frac{\lambda}{1+\lambda} \mathbf{I}\right)\left(\mathbf{D}_{i} \nabla u+\mathbf{D} \nabla u_{e}\right)\right]+\chi I_{\text {ion }}(u, \boldsymbol{w})=I^{\text {app }}
$$

and, since the proportionality assumption $\mathbf{D}_{e}=\lambda \mathbf{D}_{i}$ entails $\mathbf{D}_{e} \mathbf{D}^{-1}-\frac{\lambda}{1+\lambda} \mathbf{I}=\mathbf{0}$, a formulation of the Monodomain model (see $[6,17])$ is then obtained from $(2.9)$ as

$$
\chi C_{m} \frac{\partial u}{\partial t}-\nabla \cdot \mathbf{D}_{M} \nabla u+\chi I_{\text {ion }}(u, w)=I^{\text {app }} .
$$

In absence of applied currents, the Monodomain model is accurate enough to catch the desired dynamics and effects of the action potential propagation (see [9]). Potse et al. [29] compared the action potential propagation velocities using Monodomain and Bidomain and conclude that the Monodomain solution propagates a bit slower $(2 \%)$ than the Bidomain one. However, the Bidomain model becomes necessary when current stimuli are applied in the extracellular space. Also, the Monodomain is inadequate to simulate defibrillation [37].

Coupling the second equation in (2.8) to (2.10), we obtain an Extended Monodomain system,

$$
\left\{\begin{array}{l}
\chi C_{m} \frac{\partial u_{M}}{\partial t}-\nabla \cdot \mathbf{D}_{M} \nabla u_{M}+\chi I_{\text {ion }}\left(u_{M}, \boldsymbol{w}\right)=I^{\text {app }} \\
-\nabla \cdot\left[\mathbf{D}_{i} \nabla u_{M}+\left(\mathbf{D}_{i}+\mathbf{D}_{e}\right) \nabla u_{e, M}\right]=\widetilde{I}^{\text {app }}
\end{array}\right.
$$


This has been used as preconditioner of the Bidomain problem in [16] and in the context of model adaptivity in [23]. Here (2.11) will be used as an intermediate step for devising the coupling conditions between Bidomain and Monodomain problems.

\subsection{Numerical approximation}

We give a quick glance to the numerical approximation of the models presented in the previous section. For a more detailed description see $[7,31,40]$.

\subsubsection{Time integration}

Let us assume to use a fixed time step $\Delta t$, even if time adaptive scheme have been considered as well (see e.g. $[10,28])$. We denote with superscript $n$ the variables computed at time $t^{n}=n \Delta t$. From now on we denote by $u_{B}$, the transmembrane potential computed with the Bidomain system and by $u_{M}$ the one computed by either the Monodomain or Extended Monodomain problems. Similarly, the extracellular potential computed by the Bidomain model is denoted by $u_{e, B}$. Notation $u_{e, M}$ is used for the Extended Monodomain extracellular potential.

The Bidomain equations (2.8) can be advanced in time by a semi-implicit scheme. The ionic variables $\boldsymbol{w}$ are integrated exactly in time upon an appropriate linearization around the membrane potential at the previous time step (see e.g. [28]). The time step is selected to guarantee stability to the time advancing scheme. In what follows we do not rely on a specific choice for the ionic model describing the cell membrane currents. Thus from now on we simply denote by $I_{\text {ion }}(u)$ the ionic current: in the time advancing scheme, this nonlinear term is evaluated at the previous time step. More precisely, moving from time step $t^{n}$ to $t^{n+1}$ we solve on $\Omega$

$$
\left\{\begin{array}{l}
\chi C_{m} \frac{u_{B}^{n+1}-u_{B}^{n}}{\Delta t}-\nabla \cdot\left(\frac{\lambda \mathbf{D}_{i}}{1+\lambda} \nabla u_{B}^{n+1}+\frac{\lambda \mathbf{D}_{i}-\mathbf{D}_{e}}{1+\lambda} \nabla u_{e, B}^{n+1}\right)=I^{\text {app }}-\chi I_{\text {ion }}\left(u_{B}^{n}\right) \\
-\nabla \cdot\left[\mathbf{D}_{i} \nabla u_{B}^{n+1}+\left(\mathbf{D}_{i}+\mathbf{D}_{e}\right) \nabla u_{e, B}^{n+1}\right]=\widetilde{I}^{\text {app }} \\
u_{B}^{0}(\boldsymbol{x})=u_{0}^{B}(\boldsymbol{x}) \quad u_{e, B}^{0}(\boldsymbol{x})=u_{e, 0}^{B}(\boldsymbol{x}) \\
\left.\mathbf{n}^{T} \mathbf{D}_{i}\left(\nabla u_{B}^{n+1}+\nabla u_{e, B}^{n+1}\right)\right|_{\partial \Omega}=\left.0 \quad \mathbf{n}^{T} \mathbf{D}_{e} \nabla u_{e, B}^{n+1}\right|_{\partial \Omega}=0
\end{array}\right.
$$

The time discrete Extended Monodomain model on $\Omega$ reads

$$
\left\{\begin{array}{l}
\chi C_{m} \frac{u_{M}^{n+1}-u_{M}^{n}}{\Delta t}-\nabla \cdot \mathbf{D}_{M} \nabla u_{M}^{n+1}=I^{\text {app }}-\chi I_{\text {ion }}\left(u_{M}^{n}\right) \\
-\nabla \cdot\left[\mathbf{D}_{i} \nabla u_{M}^{n+1}+\left(\mathbf{D}_{i}+\mathbf{D}_{e}\right) \nabla u_{e, M}^{n+1}\right]=\widetilde{I}^{\text {app }} \\
u_{M}^{0}(\boldsymbol{x})=u_{0}^{M}(\boldsymbol{x}) \quad u_{e, M}^{0}(\boldsymbol{x})=u_{e, 0}^{M}(\boldsymbol{x}) \\
\left.\mathbf{n}^{T} \mathbf{D}_{M} \nabla u_{M}^{n+1}\right|_{\partial \Omega}=0 \quad \mathbf{n}^{T} \mathbf{D}_{i} \nabla u_{M}^{n+1}+\left.\mathbf{n}^{T}\left(\mathbf{D}_{i}+\mathbf{D}_{e}\right) \nabla u_{e, M}^{n+1}\right|_{\partial \Omega}=0 .
\end{array}\right.
$$

Time discretization of the Monodomain problem is formally obtained by considering the equation and the conditions in (2.13) involving only $u_{M}$.

We denote $f_{B}^{n}=I^{\text {app }}-\chi I_{\text {ion }}\left(u_{B}^{n}\right)+\frac{\chi C_{m}}{\Delta t} u_{B}^{n}$ and $g=\widetilde{I}^{\text {app }}, f_{M}^{n}=I^{\text {app }}-\chi I_{\text {ion }}\left(u_{M}^{n}\right)+\frac{\chi C_{m}}{\Delta t} u_{M}^{n}$.

In the sequel, when the context is clear we will drop the time index $n+1$. 


\subsubsection{Space discretization}

We discretize in space the domain with a regular triangulation $\mathcal{T}_{h}$ and we consider a finite element space $V_{h}$, in which we will look for the approximate solutions, namely $u_{B}^{h}$ and $u_{e, B}^{h}$ in the Bidomain case, $u_{M}^{h}$ in the Monodomain case, and $u_{M}^{h}$ and $u_{e, M}^{h}$ in the Extended Monodomain one. For the numerical tests in Section 6, $V_{h}$ is the space of piecewise linear continuous functions on $\mathcal{T}_{h}$.

We denote by $\Phi=\left\{\varphi_{j}\right\}_{j=1}^{N_{h}}$ a basis for $V_{h}$, by $\mathcal{M}$ the mass matrix with entries $\mathcal{M}^{i j}=\left.\sum_{K \in \mathcal{T}_{h}}\left(\varphi_{j}, \varphi_{i}\right)\right|_{K}$, and by $\mathcal{K}_{\tau}(\tau=i, e, M)$ the stiffness matrices with $\mathcal{K}_{\tau}^{i j}=\left.\sum_{K \in \mathcal{T}_{h}}\left(\mathbf{D}_{\tau} \nabla \varphi_{j}, \nabla \varphi_{i}\right)\right|_{K}, \varphi_{i}, \varphi_{j} \in \Phi$. When solving the Bidomain system, the unknowns of the fully discrete problem are represented by vectors $\mathbf{u}_{B}$ and $\mathbf{u}_{e, B}$, storing the nodal values of $u_{B}^{h}$ and $u_{e, B}^{h}$, respectively, we let $\mathbf{f}_{B}$ and $\mathbf{g}$ denote the discretization of the forcing terms, and we set

$$
\mathbf{B}_{u u}^{M}=\frac{\chi C_{m}}{\Delta t} \mathcal{M}+\mathcal{K}_{M} \quad \mathbf{B}_{u u}^{B}=\frac{\lambda \mathcal{K}_{i}}{1+\lambda}-\mathcal{K}_{M} \quad \mathbf{B}_{u e}=\frac{\lambda \mathcal{K}_{i}}{1+\lambda}-\frac{\mathcal{K}_{e}}{1+\lambda} \quad \mathbf{B}_{e u}=\mathcal{K}_{i} \quad \mathbf{B}_{e e}=\mathcal{K}_{i}+\mathcal{K}_{e}
$$

At step $t^{n+1}$ the discrete Bidomain models solves

$$
\mathbf{B} \mathcal{U}_{B}^{n+1}=\mathbf{f}^{n}, \quad \text { where } \quad \mathbf{B}=\left[\begin{array}{ll}
\mathbf{B}_{u u}^{B}+\mathbf{B}_{u u}^{M} & \mathbf{B}_{u e} \\
\mathbf{B}_{e u} & \mathbf{B}_{e e}
\end{array}\right], \quad \mathcal{U}_{B}=\left[\begin{array}{l}
\mathbf{u}_{B} \\
\mathbf{u}_{e, B}
\end{array}\right], \quad \mathbf{f}=\left[\begin{array}{l}
\mathbf{f}_{B} \\
\mathbf{g}
\end{array}\right] .
$$

Since the Bidomain system (2.4) is degenerate, the matrix B in its discrete formulation is singular, with a kernel spanned by the constants. We thus solve (2.14) with an iterative method (GMRES) and we force a zero mean value on the extracellular potential by imposing $\mathbf{1}^{T} \mathcal{M} \mathbf{u}_{e, B}=0$. When solving the Monodomain system, the unknown vector $\mathbf{u}_{M}$ stores the nodal values of $u_{M}^{h}, \mathbf{f}_{M}$ represents the discretization of the forcing term and at each time step the discrete Monodomain model solves system,

$$
\mathbf{B}_{u u}^{M} \mathbf{u}_{M}^{n+1}=\mathbf{f}_{M}^{n}
$$

whose matrix, differently from (2.14), is symmetric and positive so its solution can be obtained with a CG solver. Finally, when solving the Extended Monodomain system, the unknown vector $\mathbf{u}_{E}$ stores the nodal values of $u_{M}^{h}$ and $u_{e, M}^{h}, \mathbf{f}_{E}$ represents the discretization of the forcing term, and, owing to (2.13), at step $t^{n+1}$ the discrete Extended Monodomain system reads

$$
\mathbf{E} \mathcal{U}_{E}^{n+1}=\mathbf{f}_{E}^{n}, \quad \text { where } \quad \mathbf{E}=\left[\begin{array}{ll}
\mathbf{B}_{u u}^{M} & \mathbf{0} \\
\mathbf{B}_{e u} & \mathbf{B}_{e e}
\end{array}\right], \quad \mathcal{U}_{E}=\left[\begin{array}{l}
\mathbf{u}_{M} \\
\mathbf{u}_{e, M}
\end{array}\right], \quad \mathbf{f}_{E}=\left[\begin{array}{l}
\mathbf{f}_{M} \\
\mathbf{g}
\end{array}\right] .
$$

System (2.16) features a singular matrix (stemming from the purely elliptic part of the Bidomain system, that is retained here). Again, due to its lack of symmetry, system (2.16) can be solved by GMRES, and a zero mean value on the extracellular potential is then imposed by forcing $\mathbf{1}^{T} \mathcal{M} \mathbf{u}_{e, M}=0$.

\section{Model Coupling}

We devote this section to devise suitable coupling conditions for the Bidomain and the Monodomain models in a domain decomposition framework (for an introduction to domain decomposition methods see for instance $[30,34,36])$. Such conditions are derived by a downscaling procedure stemming from a Bidomain/Bidomain coupling and involving a Bidomain/Extended Monodomain coupling.

\subsection{Bidomain-Bidomain coupling}

Let us first consider a domain decomposition approach for the Bidomain model. Let $\Omega=\Omega_{1} \cup \Omega_{2}, \Sigma=$ $\partial \Omega_{1} \cap \partial \Omega_{2}$, and let us denote with $u_{i}^{m}, u_{e}^{m}$, and $u^{m}=u_{i}^{m}-u_{e}^{m}(m=1,2)$ the intracellular, extracellular and transmembrane potential in $\Omega_{1}$ and $\Omega_{2}$, respectively. 
The natural interface continuity requirements on traces and fluxes of intracellular and extracellular potentials can be obtained by the Robin coupling along $\Sigma$

$$
\boldsymbol{n}_{1}^{T} \mathbf{D}_{i} \nabla u_{i}^{1} \pm \alpha u_{i}^{1}=\boldsymbol{n}_{1}^{T} \mathbf{D}_{i} \nabla u_{i}^{2} \pm \alpha u_{i}^{2}, \quad \boldsymbol{n}_{1}^{T} \mathbf{D}_{e} \nabla u_{e}^{1} \pm \beta u_{e}^{1}=\boldsymbol{n}_{1}^{T} \mathbf{D}_{e} \nabla u_{e}^{2} \pm \beta u_{e}^{2},
$$

for $\alpha, \beta>0$.

The above conditions can be recombined in terms of $u$ and $u_{e}$ in order to have viable expressions to be used in the non-symmetric case formulation of (2.9). The latter is in fact the most natural formulation for our DD problem. Linear combinations of $(3.1)$ with coefficient $\left(\frac{\lambda}{1+\lambda},-\frac{1}{1+\lambda}\right)$ and $(1,1)$ (the same ones behind the non-symmetric formulation) provide the following equivalent coupling conditions on $\Sigma$,

$$
\begin{aligned}
\boldsymbol{n}_{1}^{T} \frac{\lambda \mathbf{D}_{i}}{1+\lambda}\left(\nabla u^{1}+\nabla u_{e}^{1}\right)-\boldsymbol{n}_{1}^{T} \frac{\mathbf{D}_{e}}{1+\lambda} \nabla u_{e}^{1}+\frac{\lambda \alpha}{1+\lambda} u^{1}+\frac{\lambda \alpha-\beta}{1+\lambda} u_{e}^{1}= & \boldsymbol{n}_{1}^{T} \frac{\lambda \mathbf{D}_{i}}{1+\lambda}\left(\nabla u^{2}+\nabla u_{e}^{2}\right) \\
& -\boldsymbol{n}_{1}^{T} \frac{\mathbf{D}_{e}}{1+\lambda} \nabla u_{e}^{2}+\frac{\lambda \alpha}{1+\lambda} u^{2}+\frac{\lambda \alpha-\beta}{1+\lambda} u_{e}^{2}, \\
\boldsymbol{n}_{1}^{T} \mathbf{D}_{i}\left(\nabla u^{1}+\nabla u_{e}^{1}\right)+\boldsymbol{n}_{1}^{T} \mathbf{D}_{e} \nabla u_{e}^{1}+\alpha u^{1}+(\alpha+\beta) u_{e}^{1}= & \boldsymbol{n}_{1}^{T} \mathbf{D}_{i}\left(\nabla u^{2}+\nabla u_{e}^{2}\right)+\boldsymbol{n}_{1}^{T} \mathbf{D}_{e} \nabla u_{e}^{2}+\alpha u^{2}+(\alpha+\beta) u_{e}^{2} .
\end{aligned}
$$

Since the linear combinations providing (3.2) and (3.3) are the same that provide the non-symmetric formulation, the flux terms in the interface conditions (3.2) and (3.3) naturally appear as boundary terms in the variational formulation, ensuring well-posedness for the local subproblems in the coupling.

\subsection{Bidomain-Extended Monodomain coupling}

Let us consider the coupling between Bidomain (2.9) and the Extended Monodomain system (2.11) defined on $\Omega_{\mathrm{B}}$ and $\Omega_{\mathrm{M}}$ respectively. We denote the interface by $\Gamma=\partial \Omega_{\mathrm{B}} \cap \partial \Omega_{\mathrm{M}}$. Since we refer to the non-symmetric formulation, condition (3.2) is the natural candidate to enforce the continuity across the interfaces of the traces and the flux of the transmembrane potential. Moreover, as a consequence of the assumptions leading to the Monodomain model, we choose therein $\beta=\lambda \alpha$. More precisely, we weigh the traces of the intra- and extracellular potentials in the Robin-Robin conditions in the same way we do for obtaining the Monodomain equation. The continuity interface conditions are thus given by

$$
\boldsymbol{n}_{B}^{T} \frac{\lambda \mathbf{D}_{i}}{1+\lambda}\left(\nabla u_{B}+\nabla u_{e, B}\right)-\boldsymbol{n}_{B}^{T} \frac{\mathbf{D}_{e}}{1+\lambda} \nabla u_{e, B}+\frac{\lambda \alpha}{1+\lambda} u_{B}=\boldsymbol{n}_{B}^{T} \mathbf{D}_{M} \nabla u_{M}+\frac{\lambda \alpha}{1+\lambda} u_{M},
$$

and

$$
\begin{aligned}
\boldsymbol{n}_{B}^{T} \mathbf{D}_{i}\left(\nabla u_{B}+\nabla u_{e, B}\right)+\boldsymbol{n}_{B}^{T} \mathbf{D}_{e} \nabla u_{e, B}+\alpha u_{B}+(1+\lambda) \alpha u_{e, B} \\
\quad=\boldsymbol{n}_{B}^{T} \mathbf{D}_{i}\left(\nabla u_{M}+\nabla u_{e, M}\right)+\boldsymbol{n}_{B}^{T} \mathbf{D}_{e} \nabla u_{e, M}+\alpha u_{M}+(1+\lambda) \alpha u_{e, M} .
\end{aligned}
$$

\subsection{Bidomain-Monodomain coupling}

Finally let us turn to the coupling between Bidomain (2.9) and Monodomain systems. We define $\Omega_{\mathrm{B}}, \Omega_{\mathrm{M}}$, and $\Gamma$ as in the previous section. According to the different sizes of the Bidomain and the Monodomain problem (the first features two unknowns, the latter only one), we have to impose on the interface $\Gamma$ two conditions for the solution in $\Omega_{\mathrm{B}}$ and one condition for the solution in $\Omega_{\mathrm{M}}$. This leads to a nonstandard domain decomposition, where the coupling conditions are based on (3.4) and (3.5). Owing to the non-symmetric formulation and to the presence of the sole transmembrane potential unknown $u_{M}$ in $\Omega_{\mathrm{M}}$, condition (3.4) still enforces the continuity across the interfaces of the trace and the flux of the transmembrane potential. To close the problem in $\Omega_{\mathrm{B}}$ 
we have to impose another boundary condition on $\Gamma$. Since we do not have an equation for the extracellular potential in the Monodomain region $\Omega_{\mathrm{M}}$, we devise an interface condition resorting to an approximation of the right hand side of condition (3.5). We propose to take

$$
u_{e, M}=-\frac{u_{M}}{1+\lambda}+K
$$

with $K$ a constant or, more generally, a function of time to be determined. If $\tilde{I}^{\text {app }}=0$ and $\mathbf{D}_{e}=\lambda \mathbf{D}_{i}$, this choice automatically fulfills the second equation in (2.11). In particular, if $u^{\text {rest }}$ denotes the resting potential, we choose $K=\frac{1}{1+\lambda} u^{\text {rest }}$ so that $u_{e, M}=0$ when $u_{M}=u^{\text {rest }}$. We notice that the choice of $K$ does not affect the solutions $u_{B}$ and $u_{M}$, while the extracellular potential $u_{e, B}$ is determined up to a constant depending on $K$. Substituting $u_{e, M}=-\frac{1}{1+\lambda}\left(u_{M}-u^{\text {rest }}\right)$ in (3.5), and assuming $\mathbf{D}_{e}=\lambda \mathbf{D}_{i}$ to hold in $\Omega_{\mathrm{M}}$, we get

$$
\boldsymbol{n}_{B}^{T} \mathbf{D}_{i}\left(\nabla u_{B}+\nabla u_{e, B}\right)+\boldsymbol{n}_{B}^{T} \mathbf{D}_{e} \nabla u_{e, B}+\alpha u_{B}+(1+\lambda) \alpha u_{e, B}=\alpha u^{\text {rest }} .
$$

Condition (3.6) is of radiative type on the total current $\mathbf{J}_{\mathrm{tot}}=-\mathbf{D}_{i} \nabla u_{i, B}-\mathbf{D}_{e} \nabla u_{e, B}$,

$$
\boldsymbol{n}_{B}^{T} \mathbf{D}_{i} \nabla u_{i, B}+\boldsymbol{n}_{B}^{T} \mathbf{D}_{e} \nabla u_{e, B}+\alpha u_{i, B}+\lambda \alpha u_{e, B}=\alpha u^{\text {rest }},
$$

allowing to avoid spurious wave reflections of the potentials on the artificial internal boundary introduced by the interface. Here $u_{i, B}$ denotes the intracellular potential within $\Omega_{\mathrm{B}}$.

Remark 3.1. The choice for approximating the term $u_{e, M}$ is obviously not unique. Another approximation would be provided by simply neglecting the terms in $u_{e, M}$ in the right hand side of condition (3.5) and assuming that $D_{e}=\lambda D_{i}$. In such case one gets

$$
\boldsymbol{n}_{B}^{T} \mathbf{D}_{i}\left(\nabla u_{B}+\nabla u_{e, B}\right)+\boldsymbol{n}_{B}^{T} \mathbf{D}_{e} \nabla u_{e, B}+\alpha u_{B}+(1+\lambda) \alpha u_{e, B}=\frac{1+\lambda}{\lambda} \boldsymbol{n}_{B}^{T} \mathbf{D}_{M} \nabla u_{M}+\alpha u_{M},
$$

and equation (3.8) is still imposing a boundary condition of radiative type on the extracellular current $\mathbf{J}_{e}=$ $-\mathbf{D}_{e} \nabla u_{e, B}$,

$$
\boldsymbol{n}_{B}^{T} \mathbf{D}_{e} \nabla u_{e, B}+\lambda \alpha u_{e, B}=0,
$$

avoiding spurious reflection of the extracellular potential wave on the interface. Numerical tests showed however that the choice (3.7) guarantees more accurate results. In both cases the flux terms in (3.4) and (3.8) appear naturally in the variational formulation of both the Bidomain and the Monodomain systems, ensuring well posedness to local subproblems in the coupling.

\section{An optimized Schwartz method}

By relying on the coupling conditions devised in the previous section, we propose to couple the Bidomain and the Monodomain models via an Optimized Schwarz Method. Such methods have become very popular in the last decade and are a generalization of the non-overlapping Robin-Robin algorithm proposed by Lions in [21], that ensures convergence also without relaxation. At each time step, the coupling conditions are enforced by the following additive Schwarz algorithm. 
Given $\eta_{1}^{0}=\boldsymbol{n}_{B}^{T} \mathbf{D}_{M} \nabla u_{M}^{0}+\frac{\lambda \alpha}{1+\lambda} u_{M}^{0}$ on $\Gamma$, solve for $p \geq 0$ until convergence

$$
\begin{cases}\frac{\chi C_{m}}{\Delta t} u_{B}^{p+1}-\nabla \cdot\left[\frac{\lambda \mathbf{D}_{i}}{1+\lambda} \nabla u_{B}^{p+1}+\frac{\lambda \mathbf{D}_{i}-\mathbf{D}_{e}}{1+\lambda} \nabla u_{e, B}^{p+1}\right]=f_{B} & \text { in } \Omega_{\mathrm{B}} \\ -\nabla \cdot\left[\mathbf{D}_{i} \nabla u_{B}^{p+1}+\left(\mathbf{D}_{i}+\mathbf{D}_{e}\right) \nabla u_{e, B}^{p+1}\right]=g_{B} & \text { in } \Omega_{\mathrm{B}} \\ \boldsymbol{n}_{B}^{T} \mathbf{D}_{i}\left(\nabla u_{B}^{p+1}+\nabla u_{e, B}^{p+1}\right)=0 \quad \boldsymbol{n}_{B}^{T} \mathbf{D}_{e} \nabla u_{e, B}^{p+1}=0 & \text { on } \partial \Omega_{\mathrm{B}} \cap \partial \Omega \\ \boldsymbol{n}_{B}^{T} \frac{\lambda \mathbf{D}_{i}}{1+\lambda}\left(\nabla u_{B}^{p+1}+\nabla u_{e, B}^{p+1}\right)-\boldsymbol{n}_{B}^{T} \frac{\mathbf{D}_{e}}{1+\lambda} \nabla u_{e, B}^{p+1}+\frac{\lambda \alpha}{1+\lambda} u_{B}^{p+1}=\eta_{1}^{p} & \text { on } \Gamma \\ \boldsymbol{n}_{B}^{T} \mathbf{D}_{i}\left(\nabla u_{B}^{p+1}+\nabla u_{e, B}^{p+1}\right)+\boldsymbol{n}_{B}^{T} \mathbf{D}_{e} \nabla u_{e, B}^{p+1}+\alpha u_{B}^{p+1}+(1+\lambda) \alpha u_{e, B}^{p+1}=\alpha u^{\text {rest }} & \text { on } \Gamma, \\ \eta_{2}^{p+1}=\boldsymbol{n}_{M}^{T} \frac{\lambda \mathbf{D}_{i}}{1+\lambda}\left(\nabla u_{B}^{p+1}+\nabla u_{e, B}^{p+1}\right)-\boldsymbol{n}_{M}^{T} \frac{\mathbf{D}_{e}}{1+\lambda} \nabla u_{e, B}^{p+1}+\frac{\lambda \alpha}{1+\lambda} u_{B}^{p+1} & \text { on } \Gamma, \\ { }_{C_{m}} n_{M}^{p+1}-\nabla \cdot \mathbf{D}_{M} \nabla u_{M}^{p+1}=f_{M} & \text { in } \Omega_{\mathrm{M}} \\ \boldsymbol{n}_{M}^{T} \mathbf{D}_{M} \nabla u_{M}^{p+1}=0 & \\ \boldsymbol{n}_{M}^{T} \mathbf{D}_{M} \nabla u_{M}^{p+1}+\frac{\lambda \alpha}{1+\lambda} u_{M}^{p+1}=\eta_{2}^{p+1} & \text { on } \partial \Omega_{\mathrm{M}} \cap \partial \Omega\end{cases}
$$

In the present form the Optimized Schwarz Algorithm is sequential, however it can be easily parallelized by choosing $\eta_{2}^{p}$ in the right hand side of the last equation in (4.3). In this case, an initial datum $\eta_{2}^{0}$ needs to be assigned as well. The above algorithm is described for a generic parameter $\lambda$ : following [9], in the sequel we choose $\lambda=\frac{\sigma_{e}^{l}}{\sigma_{i}^{l}}$.

\section{Algebraic formulation}

For sake of completeness, we outline here the discrete formulation of the Optimized Schwarz Algorithm. Let $\Omega=\Omega_{\mathrm{B}} \cup \Omega_{\mathrm{M}}$, and $\Gamma=\partial \Omega_{\mathrm{B}} \cap \partial \Omega_{\mathrm{M}}$. We still denote with $\mathbf{B}$ the matrix arising from the discretization of the Bidomain region $\Omega_{\mathrm{B}}$, and with $\mathbf{M}$ the matrix $\mathbf{B}_{u u}^{M}$ computed on the triangulation of $\Omega_{\mathrm{M}}$. We introduce the mass matrix on the interface $\boldsymbol{M}_{\Gamma}$, and the restriction matrices $\boldsymbol{R}_{B, \Gamma}, \boldsymbol{R}_{M, \Gamma}$, acting from $\Omega_{\mathrm{B}}$ and $\Omega_{\mathrm{M}}$ to the interface $\Gamma$, respectively.

At the finite dimensional level, given $\boldsymbol{\eta}_{1}^{p}=\boldsymbol{R}_{M, \Gamma}\left(\mathbf{f}_{M}-\mathbf{M} \mathbf{u}_{M}^{p}\right)+\frac{\sigma_{e}^{l}}{\sigma_{i}^{l}+\sigma_{e}^{l}} \alpha \boldsymbol{M}_{\Gamma} \boldsymbol{R}_{M, \Gamma} \mathbf{u}_{M}^{p}$, the $(p+1)$-th iteration of the Optimized Schwarz Algorithm reads

$$
\left(\mathbf{B}+\left[\begin{array}{ll}
\frac{\sigma_{e}^{l}}{\sigma_{i}^{l}+\sigma_{e}^{l}} \alpha \boldsymbol{R}_{B, \Gamma}^{T} \boldsymbol{M}_{\Gamma} \boldsymbol{R}_{B, \Gamma} & \mathbf{0} \\
\alpha \boldsymbol{R}_{B, \Gamma}^{T} \boldsymbol{M}_{\Gamma} \boldsymbol{R}_{B, \Gamma} & \frac{\sigma_{i}^{l}+\sigma_{e}^{l}}{\sigma_{i}^{l}} \alpha \boldsymbol{R}_{B, \Gamma}^{T} \boldsymbol{M}_{\Gamma} \boldsymbol{R}_{B, \Gamma}
\end{array}\right]\right)\left[\begin{array}{c}
\mathbf{u}_{B}^{p+1} \\
\mathbf{u}_{e, B}^{p+1}
\end{array}\right]=\left[\begin{array}{c}
\mathbf{f}+\boldsymbol{R}_{B, \Gamma}^{T} \boldsymbol{\eta}_{1}^{p} \\
\mathbf{g}+\alpha \mathbf{u}^{\text {rest }}
\end{array}\right]
$$




$$
\begin{gathered}
\boldsymbol{\eta}_{2}^{p+1}=\boldsymbol{R}_{B, \Gamma}\left(\mathbf{f}-\left(\mathbf{B}_{u u}^{B}+\mathbf{B}_{u u}^{M}\right) \mathbf{u}_{B}^{p+1}-\mathbf{B}_{u e} \mathbf{u}_{e, B}^{p+1}\right)+\frac{\sigma_{e}^{l}}{\sigma_{i}^{l}+\sigma_{e}^{l}} \alpha \boldsymbol{M}_{\Gamma} \boldsymbol{R}_{B, \Gamma} \mathbf{u}_{B}^{p+1} \\
\left(\mathbf{M}+\frac{\sigma_{e}^{l}}{\sigma_{i}^{l}+\sigma_{e}^{l}} \alpha \boldsymbol{R}_{M, \Gamma}^{T} \boldsymbol{M}_{\Gamma} \boldsymbol{R}_{M, \Gamma}\right) \mathbf{u}_{M}^{p+1}=\mathbf{f}_{M}+\boldsymbol{R}_{M, \Gamma}^{T} \boldsymbol{\eta}_{2}^{p+1} \\
\boldsymbol{\eta}_{1}^{p+1}=\boldsymbol{R}_{M, \Gamma}\left(\mathbf{f}_{M}-\mathbf{M} \mathbf{u}_{M}^{p+1}\right)+\frac{\sigma_{e}^{l}}{\sigma_{i}^{l}+\sigma_{e}^{l}} \alpha \boldsymbol{M}_{\Gamma} \boldsymbol{R}_{M, \Gamma} \mathbf{u}_{M}^{p+1}
\end{gathered}
$$

\subsection{Convergence analysis}

We analyze here the convergence properties of the proposed algorithm via Fourier analysis, and to this aim we consider throughout this section the problem on the infinite domain $\Omega=\mathbb{R}^{3}$, decomposed into

$$
\Omega_{\mathrm{B}}=(-\infty, 0) \times \mathbb{R}^{2}, \quad \Omega_{\mathrm{M}}=(0, \infty) \times \mathbb{R}^{2},
$$

where the interface is $\Gamma=\{0\} \times \mathbb{R}^{2}$. We disregard here the boundary conditions and require the solutions to be bounded at infinity. We assume axial symmetry of the fibers, and the longitudinal axis of the fibers (the principal direction of the action potential propagation) to be orthogonal to the interface $\Gamma$, so that the diffusion tensors are given by

$$
\mathbf{D}_{i}=\left[\begin{array}{cc}
\sigma_{i}^{l} & \\
& \sigma_{i}^{t} \mathbf{I}_{2}
\end{array}\right] \quad \mathbf{D}_{e}=\left[\begin{array}{cc}
\sigma_{e}^{l} & \\
& \sigma_{e}^{t} \mathbf{I}_{2}
\end{array}\right] \quad \mathbf{D}_{M}=\left[\begin{array}{cc}
\frac{\sigma_{i}^{l} \sigma_{e}^{l}}{\sigma_{i}^{l}+\sigma_{e}^{l}} & \\
& \frac{\sigma_{i}^{t} \sigma_{e}^{t}}{\sigma_{i}^{t}+\sigma_{e}^{t}} \mathbf{I}_{2}
\end{array}\right],
$$

being $\mathbf{I}_{2}$ the $2 \times 2$ identity matrix.

Fourier transform in the $y$ and $z$ directions is defined for any $w(x, y, z) \in L^{2}\left(\mathbb{R}^{3}\right)$ as

$$
\mathcal{F}: w(x, y, z) \mapsto \widehat{w}\left(x, k_{y}, k_{z}\right)=\iint_{\mathbb{R}^{2}} \mathrm{e}^{-\mathrm{i}\left(k_{y} y+k_{z} z\right)} u(x, y, z) \mathrm{d} y \mathrm{~d} z,
$$

where we denote by $k_{y}$ and $k_{z}$ the dual variables. We note in the sequel $\boldsymbol{k}^{2}=k_{y}^{2}+k_{z}^{2}$, and we can quantify the error, in the frequency space, between the iterates $\widehat{\eta}_{1}^{p}(\boldsymbol{k})$ and $\widehat{\eta}_{2}^{p}(\boldsymbol{k})$ and the exact interface variables $\widehat{\eta}_{1}(\boldsymbol{k})$ and $\widehat{\eta}_{2}(\boldsymbol{k})$. This allows us to define the reduction factor of the Schwarz algorithm at the $(p+1)$-th iteration as

$$
\rho^{p+1}(\boldsymbol{k}, \alpha)=\frac{\left|\widehat{\eta}_{1}^{p+1}(\boldsymbol{k})-\widehat{\eta}_{1}(\boldsymbol{k})\right|}{\left|\widehat{\eta}_{1}^{p}(\boldsymbol{k})-\widehat{\eta}_{1}(\boldsymbol{k})\right|}=\frac{\left|\widehat{\eta}_{2}^{p+1}(\boldsymbol{k})-\widehat{\eta}_{2}(\boldsymbol{k})\right|}{\left|\widehat{\eta}_{2}^{p}(\boldsymbol{k})-\widehat{\eta}_{2}(\boldsymbol{k})\right|} .
$$

Since the time discretized problem is linear, it is enough to analyze the convergence to the zero solution in the absence of forcing terms (i.e. for $f_{B}=g=f_{M}=u^{\text {rest }}=0$ ). We first formulate the Fourier transformed Bidomain and Monodomain systems.

In the Fourier space, the Bidomain equation is given by

$$
\left[\begin{array}{cc}
\frac{\sigma_{i}^{l} \sigma_{e}^{l}}{\sigma_{i}^{l}+\sigma_{e}^{l}} & 0 \\
\sigma_{i}^{l} & \sigma_{i}^{l}+\sigma_{e}^{l}
\end{array}\right] \partial_{x x}\left[\begin{array}{c}
\widehat{u}_{B} \\
\widehat{u}_{e, B}
\end{array}\right]=\left[\begin{array}{cc}
\frac{\chi C_{m}}{\Delta t}+\frac{\sigma_{e}^{l}}{\sigma_{i}^{l}+\sigma_{e}^{l}} \sigma_{i}^{t} \boldsymbol{k}^{2} & \frac{\sigma_{e}^{l} \sigma_{i}^{t}-\sigma_{e}^{t} \sigma_{i}^{l}}{\sigma_{i}^{l}+\sigma_{e}^{l}} \boldsymbol{k}^{2} \\
\sigma_{i}^{t} \boldsymbol{k}^{2} & \left(\sigma_{i}^{t}+\sigma_{e}^{t}\right) \boldsymbol{k}^{2}
\end{array}\right]\left[\begin{array}{c}
\widehat{u}_{B} \\
\widehat{u}_{e, B}
\end{array}\right],
$$

and a simple algebra yields, for $\boldsymbol{k} \in \mathbb{R}$ and $x \leq 0$,

$$
\partial_{x x}\left[\begin{array}{l}
\widehat{u}_{B} \\
\widehat{u}_{e, B}
\end{array}\right]=A(\boldsymbol{k})\left[\begin{array}{c}
\widehat{u}_{B} \\
\widehat{u}_{e, B}
\end{array}\right], \quad A(\boldsymbol{k})=\left[\begin{array}{ll}
\frac{\chi C_{m}}{\Delta t}\left(\frac{1}{\sigma_{i}^{l}}+\frac{1}{\sigma_{e}^{l}}\right)+\frac{\sigma_{i}^{t}}{\sigma_{i}^{l}} \boldsymbol{k}^{2} & \left(\frac{\sigma_{i}^{t}}{\sigma_{i}^{l}}-\frac{\sigma_{e}^{t}}{\sigma_{e}^{l}}\right) \boldsymbol{k}^{2} \\
-\frac{\chi C_{m}}{\Delta t} \frac{1}{\sigma_{e}^{l}} & \frac{\sigma_{e}^{t}}{\sigma_{e}^{l}} \boldsymbol{k}^{2}
\end{array}\right] .
$$


Analogously, the transformed Monodomain equation can be written, for $\boldsymbol{k} \in \mathbb{R}$ and $x \geq 0$, as

$$
\partial_{x x} \widehat{u}_{M}=\mu_{M}(\boldsymbol{k}) \widehat{u}_{M}, \quad \quad \mu_{M}(\boldsymbol{k})=\frac{\chi C_{m}}{\Delta t}\left(\frac{1}{\sigma_{i}^{l}}+\frac{1}{\sigma_{e}^{l}}\right)+\frac{\sigma_{i}^{t} \sigma_{e}^{t}}{\sigma_{i}^{t}+\sigma_{e}^{t}} \frac{\sigma_{i}^{l}+\sigma_{e}^{l}}{\sigma_{i}^{l} \sigma_{e}^{l}} \boldsymbol{k}^{2} .
$$

Proposition 4.1. For $\alpha>0$, the Optimized Schwarz Algorithm (4.1)-(4.4) converges for every initial guess, and for every Fourier mode $\boldsymbol{k}$ its reduction factor is independent of the iteration and is given by

$$
\rho(\boldsymbol{k}, \alpha)=\left|\frac{\alpha[1-\Psi(\boldsymbol{k}, \alpha)]-\sigma_{i}^{l}\left[1-\Psi(\boldsymbol{k}, \alpha) \frac{\sqrt{\eta^{-}(\boldsymbol{k})}}{\sqrt{\eta^{+}(\boldsymbol{k})}}\right]}{\alpha[1-\Psi(\boldsymbol{k}, \alpha)]+\sigma_{i}^{l}\left[1-\Psi(\boldsymbol{k}, \alpha) \frac{\sqrt{\eta^{-}(\boldsymbol{k})}}{\sqrt{\eta^{+}(\boldsymbol{k})}}\right]}\right| \cdot\left|\frac{\alpha-\sigma_{i}^{l} \sqrt{\mu_{M}(\boldsymbol{k})}}{\alpha+\sigma_{i}^{l} \sqrt{\mu_{M}(\boldsymbol{k})}}\right|,
$$

where

$$
\Psi(\boldsymbol{k}, \alpha)=\frac{\left(\sigma_{i}^{t}+\sigma_{e}^{t}\right) \boldsymbol{k}^{2}-\left(\sigma_{i}^{l}+\sigma_{e}^{l}\right) \eta^{-}(\boldsymbol{k})}{\left(\sigma_{i}^{t}+\sigma_{e}^{t}\right) \boldsymbol{k}^{2}-\left(\sigma_{i}^{l}+\sigma_{e}^{l}\right) \eta^{+}(\boldsymbol{k})} \cdot \frac{\alpha+\sigma_{i}^{l} \sqrt{\eta^{+}(\boldsymbol{k})}}{\alpha+\sigma_{i}^{l} \sqrt{\eta^{-}(\boldsymbol{k})}} \cdot
$$

Here $\eta^{ \pm}(\boldsymbol{k})$ are the eigenvalues of the $2 \times 2$ matrix $A(\boldsymbol{k})$ defined in (4.6), and $\mu_{M}(\boldsymbol{k})$ has been introduced in (4.7).

Proof. In the Fourier space the $(p+1)$-th iteration of the Optimized Schwarz Algorithm can be rewritten as follows. Given $\widehat{\eta}_{1}^{p}=\frac{\sigma_{i}^{l} \sigma_{e}^{l}}{\sigma_{i}^{l}+\sigma_{e}^{l}} \partial_{x} \widehat{u}_{M}^{p}+\frac{\sigma_{e}^{l}}{\sigma_{i}^{l}+\sigma_{e}^{l}} \alpha \widehat{u}_{M}^{p}$ on $\{\boldsymbol{k} \in \mathbb{R}, x=0\}$, solve

$$
\begin{aligned}
& \begin{cases}\partial_{x x}\left[\begin{array}{l}
\widehat{u}_{B}^{p+1} \\
\widehat{u}_{e, B}^{p+1}
\end{array}\right]=A(\boldsymbol{k})\left[\begin{array}{c}
\widehat{u}_{B}^{p+1} \\
\widehat{u}_{e, B}^{p+1}
\end{array}\right] & \boldsymbol{k} \in \mathbb{R}, x<0 \\
\frac{\sigma_{e}^{l} \sigma_{i}^{l}}{\sigma_{i}^{l}+\sigma_{e}^{l}} \partial_{x} \widehat{u}_{B}^{p+1}+\frac{\sigma_{e}^{l}}{\sigma_{i}^{l}+\sigma_{e}^{l}} \alpha \widehat{u}_{B}^{p+1}=\widehat{\eta}_{1}^{p} & \boldsymbol{k} \in \mathbb{R}, x=0 \\
\sigma_{i}^{l} \partial_{x} \widehat{u}_{B}^{p+1}+\left(\sigma_{i}^{l}+\sigma_{e}^{l}\right) \partial_{x} \widehat{u}_{e, B}^{p+1}+\alpha \widehat{u}_{B}^{p+1}+\frac{\sigma_{i}^{l}+\sigma_{e}^{l}}{\sigma_{i}^{l}} \alpha \widehat{u}_{e, B}^{p+1}=0 & \boldsymbol{k} \in \mathbb{R}, x=0,\end{cases} \\
& \widehat{\eta}_{2}^{p+1}=-\frac{\sigma_{e}^{l} \sigma_{i}^{l}}{\sigma_{i}^{l}+\sigma_{e}^{l}} \partial_{x} \widehat{u}_{B}^{p+1}+\frac{\sigma_{e}^{l}}{\sigma_{i}^{l}+\sigma_{e}^{l}} \alpha \widehat{u}_{B}^{p+1} \quad \boldsymbol{k} \in \mathbb{R}, x=0, \\
& \begin{cases}\partial_{x x} \widehat{u}_{M}^{p+1}=\mu_{M}(\boldsymbol{k}) \widehat{u}_{M}^{p+1} & \boldsymbol{k} \in \mathbb{R}, x>0 \\
-\frac{\sigma_{i}^{l} \sigma_{e}^{l}}{\sigma_{i}^{l}+\sigma_{e}^{l}} \partial_{x} \widehat{u}_{M}^{p+1}+\frac{\sigma_{e}^{l}}{\sigma_{i}^{l}+\sigma_{e}^{l}} \alpha \widehat{u}_{M}^{p+1}=\widehat{\eta}_{2}^{p+1} & \boldsymbol{k} \in \mathbb{R}, x=0,\end{cases} \\
& \widehat{\eta}_{1}^{p+1}=\frac{\sigma_{i}^{l} \sigma_{e}^{l}}{\sigma_{i}^{l}+\sigma_{e}^{l}} \partial_{x} \widehat{u}_{M}^{p+1}+\frac{\sigma_{e}^{l}}{\sigma_{i}^{l}+\sigma_{e}^{l}} \alpha \widehat{u}_{M}^{p+1} \quad \boldsymbol{k} \in \mathbb{R}, x=0 .
\end{aligned}
$$

Set $\widehat{\boldsymbol{u}}^{p+1}=\left(\widehat{u}_{B}^{p+1}, \widehat{u}_{e, B}^{p+1}\right)^{T}$. We reduce the Bidomain equation in (4.10) to a first order system by introducing the auxiliary variables $\widehat{\boldsymbol{z}}^{p+1}=\left(\widehat{z}^{p+1}, \widehat{z}_{e, B}^{p+1}\right)^{T}=\partial_{x} \widehat{\boldsymbol{u}}^{p+1}$ and solving system

$$
\frac{\partial}{\partial x}\left[\begin{array}{l}
\widehat{\boldsymbol{u}}^{p+1} \\
\widehat{\boldsymbol{z}}^{p+1}
\end{array}\right]=\left[\begin{array}{ll}
O_{2} & I_{2} \\
A(\boldsymbol{k}) & O_{2}
\end{array}\right]\left[\begin{array}{l}
\widehat{\boldsymbol{u}}^{p+1} \\
\widehat{\boldsymbol{z}}^{p+1}
\end{array}\right]=\mathcal{A}\left[\begin{array}{c}
\widehat{\boldsymbol{u}}^{p+1} \\
\widehat{\boldsymbol{z}}^{p+1}
\end{array}\right] .
$$

The eigenvalues of $\mathcal{A}$ satisfy

$$
\xi^{4}-\operatorname{tr} A(\boldsymbol{k}) \xi^{2}+\operatorname{det} A(\boldsymbol{k})=0,
$$


so that (remember that $\eta^{ \pm}(\boldsymbol{k})$ are the eigenvalues of $A(\boldsymbol{k})$ ), we have

$$
\xi_{1}(\boldsymbol{k})=\sqrt{\eta^{+}(\boldsymbol{k})} \quad \xi_{2}(\boldsymbol{k})=-\sqrt{\eta^{+}(\boldsymbol{k})} \quad \xi_{3}(\boldsymbol{k})=\sqrt{\eta^{-}(\boldsymbol{k})} \quad \xi_{4}(\boldsymbol{k})=-\sqrt{\eta^{-}(\boldsymbol{k})} .
$$

The solution of (4.14) is given by

$$
\left[\begin{array}{c}
\widehat{\boldsymbol{u}}^{p+1}(x, \boldsymbol{k}) \\
\widehat{\boldsymbol{z}}^{p+1}(x, \boldsymbol{k})
\end{array}\right]=\sum_{j=1}^{4} \beta_{j}^{p+1} \boldsymbol{w}_{j}(\boldsymbol{k}) e^{\xi_{j}(\boldsymbol{k}) x},
$$

where $\boldsymbol{w}_{j}(\boldsymbol{k})$ denote the eigenvector associated to $\xi_{j}(\boldsymbol{k})(j=1, \ldots, 4)$.

Observe that $\operatorname{det} A(\boldsymbol{k})=\frac{\boldsymbol{k}^{2}}{\sigma_{i}^{l} \sigma_{e}^{l}}\left[\frac{\chi C_{m}}{\Delta t}\left(\sigma_{i}^{t}+\sigma_{e}^{t}\right)+\sigma_{i}^{t} \sigma_{e}^{t} \boldsymbol{k}^{2}\right]>0$ and

$$
[\operatorname{tr} A(\boldsymbol{k})]^{2}-4 \operatorname{det} A(\boldsymbol{k})=\left[\frac{1}{\sigma_{i}^{l}}\left(\frac{\chi C_{m}}{\Delta t}+\sigma_{i}^{t} \boldsymbol{k}^{2}\right)-\frac{1}{\sigma_{e}^{l}}\left(\frac{\chi C_{m}}{\Delta t}+\sigma_{e}^{t} \boldsymbol{k}^{2}\right)\right]^{2}+\frac{4}{\sigma_{i}^{l} \sigma_{e}^{l}}\left[\frac{\chi C_{m}}{\Delta t}\right]^{2}>0 .
$$

As a consequence, both eigenvalues $\eta^{ \pm}(\boldsymbol{k})$ are real positive, and are given by

$$
\begin{aligned}
\eta^{ \pm}(\boldsymbol{k})= & \frac{1}{2}\left[\frac{1}{\sigma_{i}^{l}}\left(\frac{\chi C_{m}}{\Delta t}+\sigma_{i}^{t} \boldsymbol{k}^{2}\right)+\frac{1}{\sigma_{e}^{l}}\left(\frac{\chi C_{m}}{\Delta t}+\sigma_{e}^{t} \boldsymbol{k}^{2}\right)\right] \\
& \pm \frac{1}{2} \sqrt{\left[\frac{1}{\sigma_{i}^{l}}\left(\frac{\chi C_{m}}{\Delta t}+\sigma_{i}^{t} \boldsymbol{k}^{2}\right)-\frac{1}{\sigma_{e}^{l}}\left(\frac{\chi C_{m}}{\Delta t}+\sigma_{e}^{t} \boldsymbol{k}^{2}\right)\right]^{2}+\frac{4}{\sigma_{i}^{l} \sigma_{e}^{l}}\left[\frac{\chi C_{m}}{\Delta t}\right]^{2}} .
\end{aligned}
$$

The condition at infinity excludes growing solutions as $x \rightarrow-\infty$, thus it has to be $\beta_{2}^{p+1}=\beta_{4}^{p+1}=0$. The eigenvectors associated with $\sqrt{\eta^{+}(\boldsymbol{k})}$ and $\sqrt{\eta^{-}(\boldsymbol{k})}$ are

$$
\boldsymbol{w}_{1}(\boldsymbol{k})=\left[\begin{array}{c}
A_{12}(\boldsymbol{k}) \\
\eta^{+}(\boldsymbol{k})-A_{11}(\boldsymbol{k}) \\
A_{12}(\boldsymbol{k}) \sqrt{\eta^{+}(\boldsymbol{k})} \\
\left(\eta^{+}(\boldsymbol{k})-A_{11}(\boldsymbol{k})\right) \sqrt{\eta^{+}(\boldsymbol{k})}
\end{array}\right] \quad \boldsymbol{w}_{3}(\boldsymbol{k})=\left[\begin{array}{c}
A_{12}(\boldsymbol{k}) \\
\eta^{-}(\boldsymbol{k})-A_{11}(\boldsymbol{k}) \\
A_{12}(\boldsymbol{k}) \sqrt{\eta^{-}(\boldsymbol{k})} \\
\left(\eta^{-}(\boldsymbol{k})-A_{11}(\boldsymbol{k})\right) \sqrt{\eta^{-}(\boldsymbol{k})}
\end{array}\right] .
$$

The general solution in $\Omega_{\mathrm{B}}$ at the $(p+1)$-th iteration is thus given by

$$
\left[\begin{array}{c}
\widehat{u}_{B}^{p+1} \\
\widehat{u}_{e, B}^{p+1}
\end{array}\right]=\beta_{1}^{p+1}\left[\begin{array}{c}
A_{12}(\boldsymbol{k}) \\
\eta^{+}(\boldsymbol{k})-A_{11}(\boldsymbol{k})
\end{array}\right] \mathrm{e}^{\sqrt{\eta^{+}(\boldsymbol{k})} x}+\beta_{3}^{p+1}\left[\begin{array}{c}
A_{12}(\boldsymbol{k}) \\
\eta^{-}(\boldsymbol{k})-A_{11}(\boldsymbol{k})
\end{array}\right] \mathrm{e}^{\sqrt{\eta^{-}(\boldsymbol{k})} x},
$$

where $\beta_{1}^{p+1}$ and $\beta_{3}^{p+1}$ are determined by the two boundary conditions on the interface $\{x=0\}$. From (4.6) and (4.15) we have

$$
\eta^{+}(\boldsymbol{k})-A_{11}(\boldsymbol{k})=-\eta^{-}(\boldsymbol{k})+\frac{\sigma_{e}^{t}}{\sigma_{e}^{l}} \boldsymbol{k}^{2} \quad \eta^{-}(\boldsymbol{k})-A_{11}(\boldsymbol{k})=-\eta^{+}(\boldsymbol{k})+\frac{\sigma_{e}^{t}}{\sigma_{e}^{l}} \boldsymbol{k}^{2},
$$

and the second interface condition in (4.10) entails

$$
\beta_{3}^{p+1}=-\frac{\left(\sigma_{i}^{t}+\sigma_{e}^{t}\right) \boldsymbol{k}^{2}-\left(\sigma_{i}^{l}+\sigma_{e}^{l}\right) \eta^{-}(\boldsymbol{k})}{\left(\sigma_{i}^{t}+\sigma_{e}^{t}\right) \boldsymbol{k}^{2}-\left(\sigma_{i}^{l}+\sigma_{e}^{l}\right) \eta^{+}(\boldsymbol{k})} \cdot \frac{\alpha+\sigma_{i}^{l} \sqrt{\eta^{+}(\boldsymbol{k})}}{\alpha+\sigma_{i}^{l} \sqrt{\eta^{-}(\boldsymbol{k})}} \beta_{1}^{p+1}=-\Psi(\boldsymbol{k}, \alpha) \beta_{1}^{p+1} .
$$

Thus

$$
\left[\begin{array}{c}
\widehat{u}_{B}^{p+1}(x, \boldsymbol{k}) \\
\widehat{u}_{e, M}^{p+1}(x, \boldsymbol{k})
\end{array}\right]=\beta_{1}^{p+1}\left(\left[\begin{array}{c}
A_{12}(\boldsymbol{k}) \\
\eta^{+}(\boldsymbol{k})-A_{11}(\boldsymbol{k})
\end{array}\right] \mathrm{e}^{\sqrt{\eta^{+}(\boldsymbol{k})} x}-\Psi(\boldsymbol{k}, \alpha)\left[\begin{array}{c}
A_{12}(\boldsymbol{k}) \\
\eta^{-}(\boldsymbol{k})-A_{11}(\boldsymbol{k})
\end{array}\right] \mathrm{e}^{\sqrt{\eta^{-}(\boldsymbol{k})} x}\right) .
$$

The value of $\beta_{1}^{p+1}$ is then uniquely determined by the coupling condition in $x=0$. 
Concerning domain $\Omega_{\mathrm{M}}$, the solution at the ( $\left.p+1\right)$-th iteration, owing to (4.12), is given by

$$
\widehat{u}_{M}^{p+1}(x, \boldsymbol{k})=\gamma_{1}^{p+1} \mathrm{e}^{-\sqrt{\mu_{M}(\boldsymbol{k})} x}+\gamma_{2}^{p+1} \mathrm{e}^{\sqrt{\mu_{M}(\boldsymbol{k})} x} .
$$

The boundedness condition at infinity entails $\gamma_{2}^{p+1}=0$, thus we have

$$
\widehat{u}_{M}^{p+1}(x, \boldsymbol{k})=\gamma_{1}^{p+1} \mathrm{e}^{-\sqrt{\mu_{M}(\boldsymbol{k})} x},
$$

and the value of $\gamma_{1}^{p+1}$ is uniquely recovered from the interface conditions at $x=0$.

Gathering together (4.11), (4.13), (4.17), and (4.18), we get

$$
\begin{array}{ll}
\frac{\sigma_{e}^{l}}{\sigma_{i}^{l}+\sigma_{e}^{l}} B_{1, B}(\boldsymbol{k}, \alpha) \beta_{1}^{p+1}=\widehat{\eta}_{1}^{p}(\boldsymbol{k}) & \widehat{\eta}_{2}^{p+1}(\boldsymbol{k})=\frac{\sigma_{e}^{l}}{\sigma_{i}^{l}+\sigma_{e}^{l}} M_{1, B}(\boldsymbol{k}, \alpha) \beta_{1}^{p+1}=\frac{M_{1, B}(\boldsymbol{k}, \alpha)}{B_{1, B}(\boldsymbol{k}, \alpha)} \widehat{\eta}_{1}^{p}(\boldsymbol{k}) \\
\frac{\sigma_{e}^{l}}{\sigma_{i}^{l}+\sigma_{e}^{l}} M_{1, M}(\boldsymbol{k}, \alpha) \gamma_{1}^{p+1}=\widehat{\eta}_{2}^{p+1}(\boldsymbol{k}) & \widehat{\eta}_{1}^{p+1}(\boldsymbol{k})=\frac{\sigma_{e}^{l}}{\sigma_{i}^{l}+\sigma_{e}^{l}} B_{1, M}(\boldsymbol{k}, \alpha) \gamma_{1}^{p+1}=\frac{B_{1, M}(\boldsymbol{k}, \alpha)}{M_{1, M}(\boldsymbol{k}, \alpha)} \widehat{\eta}_{2}^{p+1}(\boldsymbol{k}),
\end{array}
$$

where

$$
\begin{aligned}
& B_{1, B}(\boldsymbol{k}, \alpha)=A_{12}(\boldsymbol{k})\left(\alpha[1-\Psi(\boldsymbol{k}, \alpha)]+\sigma_{i}^{l}\left[\sqrt{\eta^{+}(\boldsymbol{k})}-\Psi(\boldsymbol{k}, \alpha) \sqrt{\eta^{-}(\boldsymbol{k})}\right]\right) \\
& B_{1, M}(\boldsymbol{k}, \alpha)=\alpha-\sigma_{i}^{l} \sqrt{\mu_{M}(\boldsymbol{k})} \\
& M_{1, B}(\boldsymbol{k}, \alpha)=A_{12}(\boldsymbol{k})\left(\alpha[1-\Psi(\boldsymbol{k}, \alpha)]-\sigma_{i}^{l}\left[\sqrt{\eta^{+}(\boldsymbol{k})}-\Psi(\boldsymbol{k}, \alpha) \sqrt{\eta^{-}(\boldsymbol{k})}\right]\right) \\
& M_{1, M}(\boldsymbol{k}, \alpha)=\alpha+\sigma_{i}^{l} \sqrt{\mu_{M}(\boldsymbol{k})} .
\end{aligned}
$$

From (4.19) we have

$$
\widehat{\eta}_{1}^{p+1}(\boldsymbol{k})=\frac{B_{1, M}(\boldsymbol{k}, \alpha)}{B_{1, B}(\boldsymbol{k}, \alpha)} \cdot \frac{M_{1, B}(\boldsymbol{k}, \alpha)}{M_{1, M}(\boldsymbol{k}, \alpha)} \widehat{\eta}_{1}^{p}(\boldsymbol{k})=\left[\frac{B_{1, M}(\boldsymbol{k}, \alpha)}{M_{1, M}(\boldsymbol{k}, \alpha)} \cdot \frac{M_{1, B}(\boldsymbol{k}, \alpha)}{B_{1, B}(\boldsymbol{k}, \alpha)}\right]^{p+1} \widehat{\eta}_{1}^{0}(\boldsymbol{k}),
$$

being $\widehat{\eta}_{1}^{0}(\boldsymbol{k})$ given. The reduction factor of the Schwarz Algorithm is thus independent of the iteration $p$ and for any Fourier mode $\boldsymbol{k}$ is given by

$$
\rho(\boldsymbol{k}, \alpha)=\left|\frac{B_{1, M}(\boldsymbol{k}, \alpha)}{M_{1, M}(\boldsymbol{k}, \alpha)} \cdot \frac{M_{1, B}(\boldsymbol{k}, \alpha)}{B_{1, B}(\boldsymbol{k}, \alpha)}\right|,
$$

which is (4.8). The Schwarz Algorithm (4.1)-(4.4) is convergent for every Fourier mode if $\rho(\boldsymbol{k}, \alpha)<1, \forall \boldsymbol{k} \in \mathbb{R}$. Being $\alpha>0$, it is enough to show that, $\forall \boldsymbol{k} \in \mathbb{R}, \Psi(\boldsymbol{k}, \alpha) \leq 0$. The second factor in (4.9) is positive. To prove that the first factor is non-positive we prove the equivalent condition

$$
\eta^{-}(\boldsymbol{k}) \leq \frac{\sigma_{i}^{t}+\sigma_{e}^{t}}{\sigma_{i}^{l}+\sigma_{e}^{l}} \boldsymbol{k}^{2}<\eta^{+}(\boldsymbol{k}) \quad \forall \boldsymbol{k} \in \mathbb{R} .
$$

For $\boldsymbol{k}=0$, inequality (4.20) is easily satisfied. For any $\boldsymbol{k}>0$, let

$$
\begin{gathered}
a_{1}=\frac{\sigma_{e}^{t}}{\sigma_{e}^{l}} \boldsymbol{k}^{2}, \quad b_{1}=\frac{\sigma_{i}^{t}}{\sigma_{i}^{l}} \boldsymbol{k}^{2}, \quad c=\frac{1}{\sqrt{\sigma_{i}^{l} \sigma_{e}^{l}}} \frac{\chi C_{m}}{\Delta t}, \quad d=\frac{\sigma_{i}^{t}+\sigma_{e}^{t}}{\sigma_{i}^{l}+\sigma_{e}^{l}} \boldsymbol{k}^{2}, \\
a=a_{1}+\sqrt{\frac{\sigma_{i}^{l}}{\sigma_{e}^{l}}} c=\frac{1}{\sigma_{e}^{l}}\left(\frac{\chi C_{m}}{\Delta t}+\sigma_{e}^{t} \boldsymbol{k}^{2}\right), \quad b=b_{1}+\sqrt{\frac{\sigma_{e}^{l}}{\sigma_{i}^{l}}} c=\frac{1}{\sigma_{i}^{l}}\left(\frac{\chi C_{m}}{\Delta t}+\sigma_{i}^{t} \boldsymbol{k}^{2}\right),
\end{gathered}
$$


so that

$$
\eta^{ \pm}=\frac{1}{2}(a+b) \pm \frac{1}{2} \sqrt{(a-b)^{2}+4 c^{2}} .
$$

Assuming without loss of generality $\sigma_{e}^{t} \sigma_{i}^{l} \geq \sigma_{e}^{l} \sigma_{i}^{t}$ (otherwise swap the definitions of $a$ and $b$ ), the following relations hold

$$
a_{1} \geq d \geq b_{1}, \quad a>d, \quad \sqrt{\frac{\sigma_{e}^{l}}{\sigma_{i}^{l}}}\left(a_{1}-d\right)=\sqrt{\frac{\sigma_{i}^{l}}{\sigma_{e}^{l}}}\left(d-b_{1}\right) .
$$

Then, on the one hand, we have

$$
\eta^{+}-d=\frac{1}{2}\left(a+b-2 d+\sqrt{(a-b)^{2}+4 c^{2}}\right) \geq \frac{1}{2}(a+b-2 d+|a-b|) \geq a-d>0 .
$$

On the other hand

$$
\left(\eta^{-}-d\right)\left(\eta^{+}-d\right)=\frac{1}{4}(a+b-2 d)^{2}-\frac{1}{4}(a-b)^{2}-c^{2}=-(a-d)(d-b)-c^{2},
$$

and, owing to (4.21) and (4.23)

$$
\left(\eta^{-}-d\right)\left(\eta^{+}-d\right)=-\left(d-b_{1}\right)\left(a_{1}-d\right)+c\left[\sqrt{\frac{\sigma_{e}^{l}}{\sigma_{i}^{l}}}\left(a_{1}-d\right)-\sqrt{\frac{\sigma_{i}^{l}}{\sigma_{e}^{l}}}\left(d-b_{1}\right)\right]=-\left(d-b_{1}\right)\left(a_{1}-d\right) \leq 0 .
$$

Thus, being $\eta^{+}-d>0$, we can infer $\eta^{-}-d \leq 0$, and this concludes the proof.

We point out that starting the algorithm by solving the Monodomain first would provide the same result, since

$$
\widehat{\eta}_{2}^{p+1}(\boldsymbol{k})=\frac{M_{1, B}(\boldsymbol{k}, \alpha)}{M_{1, M}(\boldsymbol{k}, \alpha)} \cdot \frac{B_{1, M}(\boldsymbol{k}, \alpha)}{B_{1, B}(\boldsymbol{k}, \alpha)} \widehat{\eta}_{2}^{p}(\boldsymbol{k}) .
$$

\subsection{Optimal Robin interface conditions and low frequency approximation}

We focus here on the choice of the parameter $\alpha$ in the Robin interface conditions. Ideally, the optimal parameter forces the reduction factor $\rho(\boldsymbol{k}, \alpha)$ to be zero, so that convergence is attained in a number of iterations equal to the number of subdomains (two, in the case at hand). Expressions for optimal parameter are obtained from $(4.8)$

$$
\alpha_{1}^{\text {exact }}(\boldsymbol{k})=\sigma_{i}^{l} \sqrt{\mu_{M}(\boldsymbol{k})} \quad \alpha_{2}^{\text {exact }}(\boldsymbol{k}): \alpha_{2}^{\text {exact }}(\boldsymbol{k})=\sigma_{i}^{l} \frac{\sqrt{\eta^{+}(\boldsymbol{k})}-\Psi\left(\boldsymbol{k}, \alpha_{2}^{\text {exact }}(\boldsymbol{k})\right) \sqrt{\eta^{-}(\boldsymbol{k})}}{\left[1-\Psi\left(\boldsymbol{k}, \alpha_{2}^{\text {exact }}(\boldsymbol{k})\right)\right] \sqrt{\eta^{+}(\boldsymbol{k})}},
$$

which unfortunately depend on the frequency $\boldsymbol{k}$. In fact, $\alpha_{1}^{\text {exact }}(\boldsymbol{k})$ and $\alpha_{2}^{\text {exact }}(\boldsymbol{k})$ are actually the symbols of two linear, pseudodifferential, operators acting along the interface. These expressions are not viable since their back transform in the space of physical variables is a nonlocal operator. We resort therefore to a different, even suboptimal, choice. We propose to approximate $\alpha_{1}^{\text {exact }}(\boldsymbol{k})$ with a constant. The standard approach in the Optimized Schwarz Methods literature (see $[1,14,15]$ ) consists in finding the values of the parameters that minimize the convergence rate over all the relevant frequencies of the problem. This amounts to solve the min-max problem

$$
\min _{\alpha, \in \mathbb{R}^{+}} \max _{\boldsymbol{k} \in\left[0, \boldsymbol{k}_{\max }\right]} \rho(\boldsymbol{k}, \alpha),
$$

where $\boldsymbol{k}_{\max }$ is the largest frequency supported by the numerical grid and is of the order $\pi / h$, where $h$ is the mesh size. Solution to (4.25) is nontrivial and it is not available at the moment. 

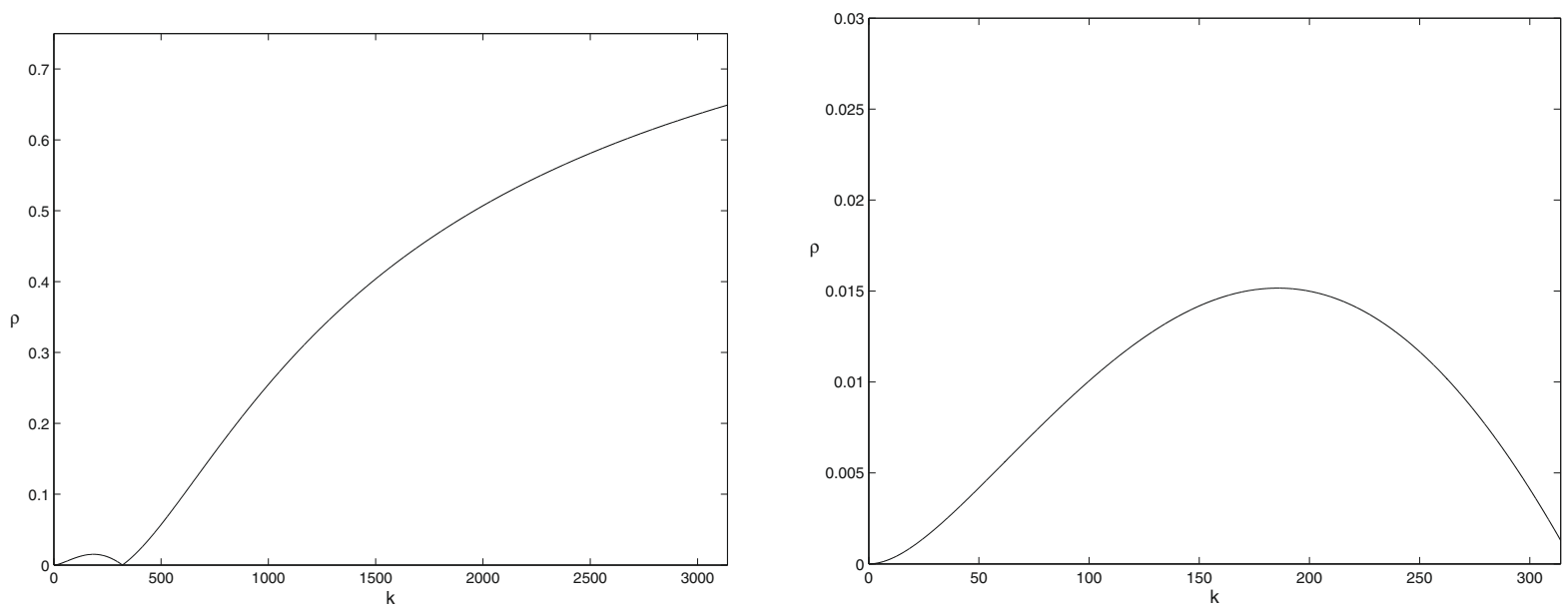

Figure 1. Reduction factor of the Optimized Schwarz Algorithm as a function of the frequency for a two domain decomposition with $\boldsymbol{k}_{\max }=1000 \pi$ (left), and with a more practical $\boldsymbol{k}_{\max }=$ $100 \pi$ (right).

Another approach is to approximate $\alpha_{1}^{\text {exact }}(\boldsymbol{k})$ with its zero-th order Taylor expansion around $\boldsymbol{k}=0$. We get

$$
\alpha^{*}=\alpha_{1}^{\text {exact }}(\mathbf{0})=\sigma_{i}^{l} \sqrt{\mu_{M}(0)}=\sigma_{i}^{l} \sqrt{\frac{\chi C_{m}}{\Delta t} \frac{\sigma_{i}^{l}+\sigma_{e}^{l}}{\sigma_{i}^{l} \sigma_{e}^{l}}} .
$$

We plot in Figure 1 the reduction factor $\rho\left(\boldsymbol{k}, \alpha^{*}\right)$ in the frequency space. Both Bidomain and Monodomain conductivities are taken from [9], the Robin parameter is the one given by (4.26), the time step is $\Delta t=0.05 \mathrm{~ms}$, and the maximal tangential frequency is $\boldsymbol{k}_{\max }=1000 \pi$ on the left, and $\boldsymbol{k}_{\max }=100 \pi$ on the right. The reduction factor of the Optimized Schwarz Algorithm tends to 1 as $\boldsymbol{k} \rightarrow \infty$. This is a common feature for this kind of algorithms, which can slow down the convergence. The reduction factor, with the choice of $\alpha^{*}$ given in (4.26), vanishes for two distinct frequencies, namely for $\boldsymbol{k}=0$, and for $\boldsymbol{k}_{0}$, the latter one being implicitly defined by

$$
\alpha^{*}\left[1-\Psi\left(\boldsymbol{k}_{0}, \alpha^{*}\right)\right] \sqrt{\eta^{+}\left(\boldsymbol{k}_{0}\right)}=\sigma_{i}^{l}\left[\sqrt{\eta^{+}\left(\boldsymbol{k}_{0}\right)}-\Psi\left(\boldsymbol{k}_{0}, \alpha^{*}\right) \sqrt{\eta^{-}\left(\boldsymbol{k}_{0}\right)}\right] .
$$

Remark 4.2. The Robin parameter introduced above has been derived in the special case of an interface orthogonal to the longitudinal axis of the fibers. The numerical tests of Section 6 show that this choice proves to be effective also in the case of a more general interface.

Remark 4.3. A similar analysis can be carried out also in the case the Optimized Schwarz Algorithm is devised with a generic parameter $\lambda$. In that case the zero-th order approximation of the exact Robin parameter is given by

$$
\alpha_{\lambda}^{*}=\frac{1+\lambda}{\lambda} \sqrt{\frac{\chi C_{m}}{\Delta t} \frac{\sigma_{i}^{l} \sigma_{e}^{l}}{\sigma_{i}^{l}+\sigma_{e}^{l}}}
$$

\section{NumERICAL RESULtS}

Numerical tests presented in this section have two different goals. In the first series of tests we analyze the performance of the Optimized Schwarz Algorithm with respect to the mesh size. The second series of tests is focused on the comparison of the solution of the coupled problem with the Bidomain solution in the whole domain. Finally, we provide a three dimensional application, in which we simulate with the propagation on a simplified left ventricular geometry of the stimulus exerted by an external pacemaker. In all the following tests 
TABLE 1. Iteration counts for various mesh sizes and different tolerances.

\begin{tabular}{|c|c|c|c|c|}
\hline$h$ & $1 / 64$ & $1 / 128$ & $1 / 256$ & $1 / 512$ \\
\hline$\varepsilon=1 \mathrm{e}-3$ & 2 & 2 & 2 & 2 \\
\hline$\varepsilon=1 \mathrm{e}-6$ & 6 & 4 & 4 & 4 \\
\hline$\varepsilon=1 \mathrm{e}-9$ & 11 & 9 & 12 & 18 \\
\hline
\end{tabular}

we use the Luo-Rudy Phase I ionic current model for ventricular cells [22], while the conductivity coefficients and the other parameters of the system are retrieved form [7]. Both Bidomain and Monodomain problems are advanced in time by the semi-implicit scheme described in Section 2.1, that linearizes the ionic current around the previous time step. In order to focus only on the performance of the Optimized Schwarz Algorithm, in all the simulations presented in this section we use a fixed time step $\Delta t=0.05 \mathrm{~ms}$, which is small enough to capture the abrupt variations in the transmembrane potential during the action potential. The ionic gating variables are integrated exactly in time after linearization, while the concentration variables are advanced in time with a Forward Euler scheme. The first series of test is run with Matlab ${ }^{\circledR} 7.5$, while the second series and the simplified ventricle are implemented in $\mathrm{LifeV}^{2}$, a $\mathrm{C}++$ finite element library that uses Trilinos packages ${ }^{3}$ to solve the linear systems.

\subsection{Influence of the mesh size and the Robin parameter}

In this first series of tests the computational domain is the rectangle $\Omega=[0,2] \times[0,1]$ decomposed in the two subdomains $\Omega_{\mathrm{B}}=[0,1] \times[0,1]$ and $\Omega_{\mathrm{M}}=[1,2] \times[0,1]$, where we solve the Bidomain and the Monodomain problems respectively. The fibers are parallel to the reference axes, with the longitudinal direction set orthogonal to the interface. Both domains are discretized by a uniform triangular grid (we denote with $h$ the mesh size), associated with $\mathbb{P}_{1}$ finite elements. Since at each time step problems to be solved are linear, we analyze here the convergence to the zero solution starting from a random value on the interface and in the absence of forcing terms. The iterative algorithm stops when the maximum between $\left\|\boldsymbol{\eta}_{1}^{p+1}-\boldsymbol{\eta}_{1}^{p}\right\|_{l^{2}(\Gamma)}$ and $\left\|\boldsymbol{\eta}_{1}^{p+1}\right\|_{l^{2}(\Gamma)}$ drops below a given tolerance $\varepsilon$. We report in Table 1 the iteration counts for different mesh sizes and different values of the stopping tolerance $\varepsilon$.

The Optimized Schwarz Algorithm is robust and effective. Two iterations are sufficient to fulfill a tolerance requirement of $\varepsilon=1 \mathrm{e}-3$, whereas four iterations suffice for a tolerance of $\varepsilon=1 \mathrm{e}-6$ independently of the mesh size. When the required tolerance is set to $\varepsilon=1 \mathrm{e}-9$, which is general fairly small for this kind of problems, a sublinear growth is observed in the iteration counts.

We then tested the optimality for the choice of $\alpha^{*}$ as the Robin parameter. We report in Figure 2 the iteration counts to reach convergence and the error after three iterations for different values of $\alpha$. Test are carried out with mesh size $h=1 / 128$, and a tolerance of $\varepsilon=1 \mathrm{e}-6$. We enlighten in both pictures the values of $\alpha^{*}$ provided by (4.26). Even if the result of an approximation, such choice appears pretty accurate with respect to the iteration counts (left) and effective with respect to the error at convergence (right).

\subsection{Convergence and accuracy of the domain decomposition solution during action potential propagation}

In this section we consider the action potential propagation on the same 2D geometry of the previous section. During the first millisecond of the simulation we apply a current stimulus $I_{i}^{\text {app }}=I_{e}^{\text {app }}=50 \mathrm{~mA} \cdot \mathrm{cm}^{-3}$ in the circle $(x-0.2)^{2}+(y-0.2)^{2} \leq(0.12)^{2}$. Figure 4 (top) shows the transmembrane potential at different time-steps computed with the Bidomain model. We solve the problem on a structured mesh with $h=1 / 64$. Simulations of larger tissue volumes typically employ a spatial resolution of between $0.1 \mathrm{~mm}$ and $0.2 \mathrm{~mm}$ typically. For example, $0.25 \mathrm{~mm}$ is an adequate resolution for the Nygren et al. model [25], while for the Fox et al. model [13],

\footnotetext{
${ }^{2}$ LifeV software, http://www.LifeV.org.

${ }^{3}$ Trilinos software, http://trilinos.sandia.gov.
} 

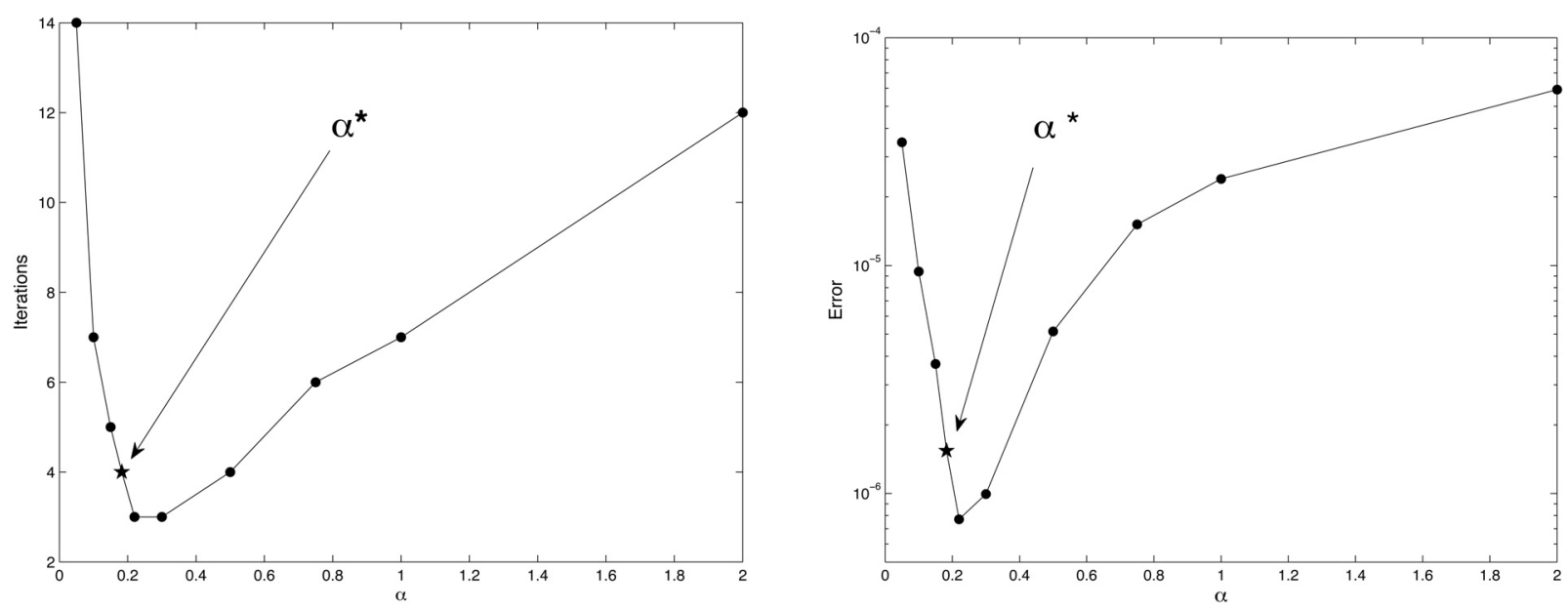

FIGURE 2. Left: iteration counts with respect to the value of the Robin parameter $\alpha$. Right: error after three iterations with respect to the value of the Robin parameter $\alpha$. $(\varepsilon=1 \mathrm{e}-6$, $h=1 / 128$.)

which has a faster upstroke, a finer resolution of $0.15 \mathrm{~mm}$ is required [5]. The linear systems are solved with the GMRES and CG algorithms provided by the library AZTEC ${ }^{4}$. In particular the Bidomain system is solved with the GMRES method preconditioned with an incomplete LU factorization, while the Monodomain equation is solved with CG method preconditioned with an incomplete Cholesky factorization. The stopping criteria used in the linear solvers are based on the Euclidean norm of the residual normalized with the Euclidean norm of the forcing term. We require the normalized residual to be less than $1 \mathrm{e}-8$.

\section{Convergence}

In Figure 3 (left) we report $\left\|u_{B}^{p}-u_{M}^{p}\right\|_{L^{\infty}(\Gamma)}$ as a function of the number of Schwarz iterations, at different time levels, chosen according to the position of the depolarization front (standard notation is adopted for Banach $L^{p}$ spaces). At $t=9 \mathrm{~ms}$ the front is approaching the interface, but it is still completely inside $\Omega_{\mathrm{B}}$, at $t=14 \mathrm{~ms}$ the front is partly in $\Omega_{\mathrm{B}}$ and partly in $\Omega_{\mathrm{M}}$, while at $t=26 \mathrm{~ms}$ the front is on his way out of $\Omega_{\mathrm{M}}$, and the interface lies in the plateau region of the action potential (top Fig. 4). The position of the depolarization front is clearly affecting the initial error, but not the convergence of the Schwarz Algorithm, which proves to be robust showing the same slope in all three conditions. For the sake of simplicity we just performed a low number of steps in the iterative framework using as initial guess the solution at the previous time step. We thus analyzed the error after two and four iterations, and we report in Figure 3 (right) the results along the time interval $[0,450] \mathrm{ms}$. Both cases show the same pattern, and the major errors are concentrated in the time frames when the depolarization front and the late repolarization wave cross the interface. This is not surprising since those are the stiff parts in the time evolution in the modelling of the action potential in a single cell.

\section{Accuracy}

We denote by $u_{\text {DD }}$ the solution obtained with the Schwarz Algorithm, with $u_{\text {Bido }}$ the solution obtained from a Bidomain simulation on the whole domain $\Omega$, and with $u_{\text {Mono }}$ the one obtained from a Monodomain simulation on the whole domain $\Omega$. In Figure 4 we report the transmembrane potential $u_{\text {Bido }}$ (top), the difference between $u_{\text {Bido }}$ and $u_{\mathrm{DD}}$, computed with two Schwarz iterations per time step (middle) and the transmembrane potential $u_{\text {Mono }}$ (bottom). Snapshots are taken at different time levels $(t=9,14,26 \mathrm{~ms}$ ). As was to be expected, error is localized in the surroundings of the depolarization front. The Monodomain model fails in the proper representation of the front velocity, being slower than in the Bidomain model [29]: this aspect is well enlightened

\footnotetext{
${ }^{4}$ AZTEC software, http://acts.nersc.gov/aztec.
} 

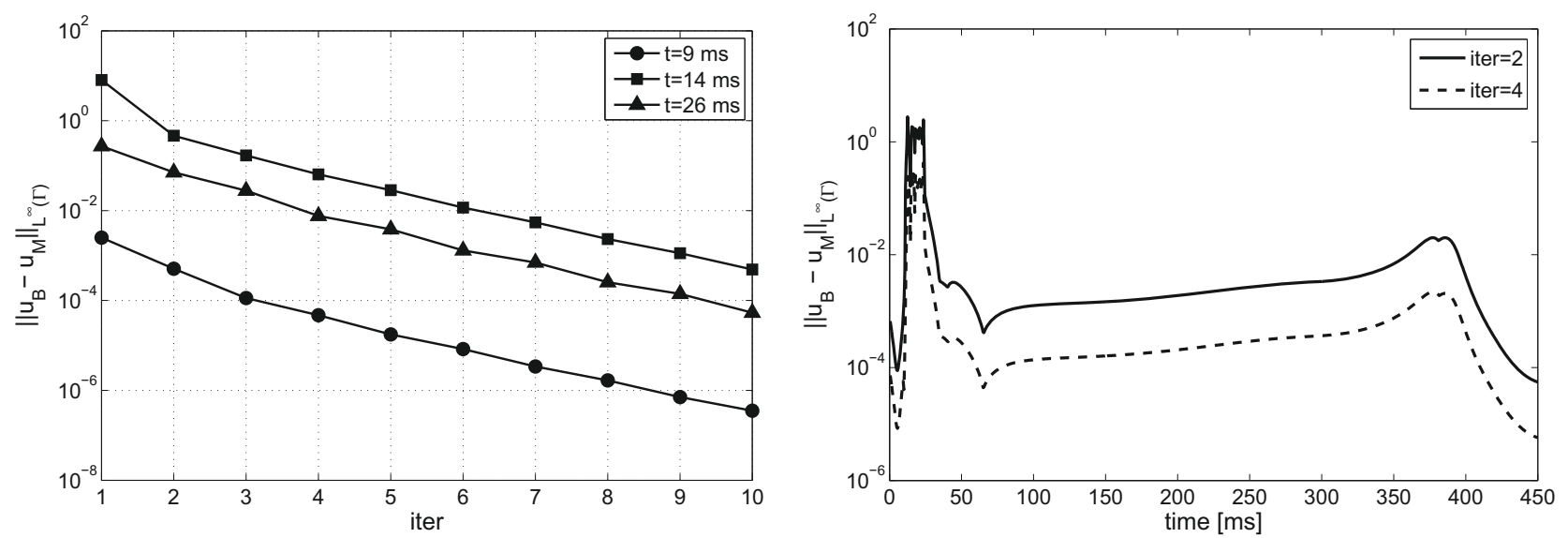

FiguRE 3. Left: $\left\|u_{B}^{p}-u_{M}^{p}\right\|_{L^{\infty}(\Gamma)}$ as a function of the number of Schwarz iterations $p$ at different time levels. Right: $\left\|u_{B}^{p}-u_{M}^{p}\right\|_{L^{\infty}(\Gamma)}$ as a function of time after two and four Schwarz iterations. Fibers orthogonal to the interface.

-85. $-55,-26 . \quad 4 . \quad 34$
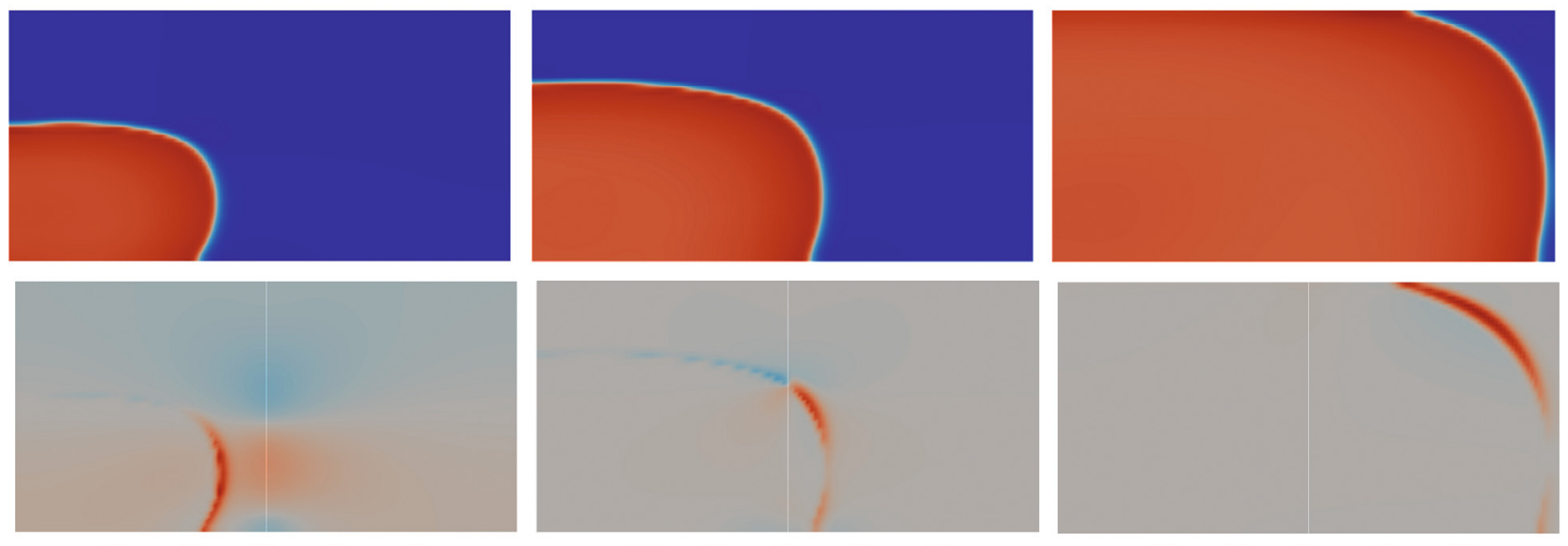

$\begin{array}{rrrrr}-1.0 & -0.5 & 0.0 & 0.5 & 1.0\end{array}$

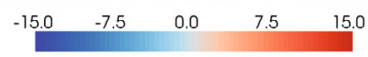

$\begin{array}{rrrr}-100 . & -50 . & 0 . & 50 .\end{array}$
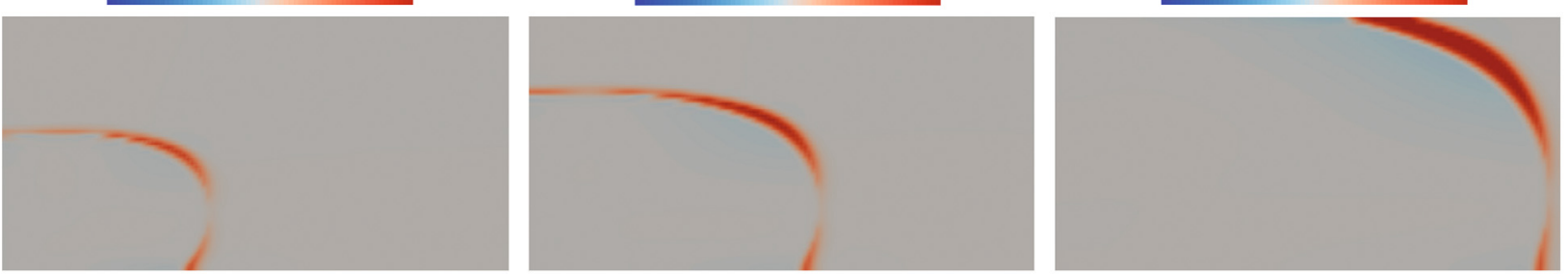

$\begin{array}{rrrr}-100 . & -50 . & 0 . & 50 . \\ \end{array}$

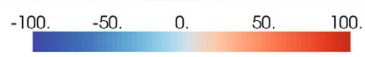

$\begin{array}{rrrr}-100 . & -50 . & 0 . & 50 .\end{array}$

FiguRE 4. Membrane potential $u_{\text {Bido }}$ (top) and errors $u_{\text {Bido }}-u_{\text {DD }}$ (with two Schwarz iterations at each time step - middle) and $u_{\text {Bido }}-u_{\text {Mono }}$ (bottom) at different time levels (left to right $t=9,14,26 \mathrm{~ms}): \Omega_{\mathrm{B}}=[0,1] \times[0,1], \Omega_{\mathrm{M}}=[1,2] \times[0,1], \theta=0$. Notice the different scales in the values. The bottom rightmost picture enlightens the different velocities of the wavefronts. 

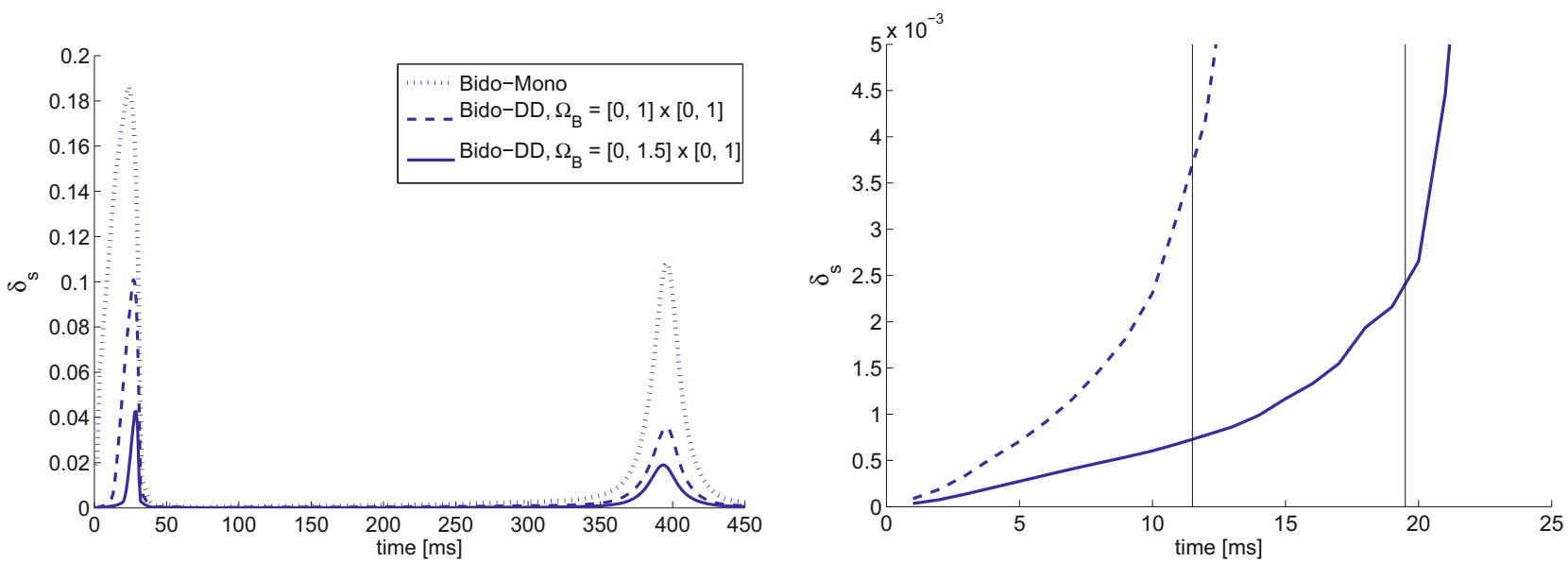

FiguRE 5. Left: relative differences in space, as a function of time, between $u_{\text {Bido }}$ and $u_{\text {Mono }}$ (dotted line), and between $u_{\mathrm{Bido}}$ and $u_{\mathrm{DD}}$ at convergence, for two different partitions of $\Omega$; $\Omega_{\mathrm{B}}=[0,1] \times[0,1]$ (dashed line) or $\Omega_{\mathrm{B}}=[0,1.5] \times[0,1]$ (solid line). Right: detail of the figure on the left. The vertical lines enlighten the times when the action potentials reach the interfaces.

by the bottom rightmost picture in Figure 4. In there, the upper boundary of the dark region identifies the Bidomain wavefront, while the lower boundary identifies the Monodomain wavefront. As a consequence, the error in the coupled simulation is actually significant as the front approaches the interface, and gets slowed down by the presence of the Monodomain region.

To quantify the error, we compute

$$
\delta_{s}=\frac{\left\|u_{\text {Bido }}-u^{*}\right\|_{L^{2}(\Omega)}}{\left\|u_{\text {Bido }}-u^{\text {rest }}\right\|_{L^{2}(\Omega)}},
$$

where $u^{*}$ is time to time the DD or the Monodomain solution. Normalization with $u_{\text {Bido }}-u^{\text {rest }}$ enhances the visualization of the errors we are interested in.

We investigate the influence of the size of the region $\Omega_{\mathrm{M}}$ on the overall accuracy. We consider two partitions of the domain $\Omega: \Omega_{\mathrm{B}}=[0,1] \times[0,1]$ and $\Omega_{\mathrm{M}}=[1,2] \times[0,1], \Omega_{\mathrm{B}}=[0,1.5] \times[0,1]$ and $\Omega_{\mathrm{M}}=[1.5,2] \times[0,1]$. In addition we consider the case $\Omega_{\mathrm{B}}=\emptyset$ and $\Omega_{\mathrm{M}}=\Omega$. In Figure 5 we illustrate the time dependence of $\delta_{s}$ for the different cases considered. From Figure 5 (left) we can appreciate the significant improvement with respect to the full Monodomain solution (dotted line). Again, the error is larger when the depolarization front and the late repolarization wave are located in $\Omega_{\mathrm{M}}$. The influence of the size of $\Omega_{\mathrm{B}}$ is visible: the error peaks at $10 \%$ when $\Omega_{\mathrm{B}}=[0,1] \times[0,1]$, and at less than $5 \%$ when $\Omega_{\mathrm{B}}=[0,1.5] \times[0,1]$. A detailed view of the early part of the simulation (Fig. 5, right) enlightens the fact that, even if the error grows as the wavefront approaches the interface (the crossing instant is put into evidence by the two vertical lines), the approximation is still very good inside $\Omega_{\mathrm{B}}$ (error peaks below $0.4 \%$ in the vicinity of the interface). This fact is promising in view of a model adaptive strategy. In this case, the final goal is to solve the Bidomain model only in those regions where it is needed, and the Monodomain model elsewhere. The problem of identifying the Bidomain and Monodomain regions have been recently addressed in [23]. In that paper the Bidomain model has been shown to be necessary where the upstroke and the recovery of the action potential occur. A model adaptive solver is supposed to be able to track the wavefront and associate it to the Bidomain model. In this scenario, it is realistic to assume that the interface between the Bidomain and the Monodomain regions is not crossed by the potential wavefront. 
TABLE 2. Relative difference in space and time between $u_{\text {Bido }}$ and $u_{\mathrm{DD}}$ (after two Schwarz iterations and at convergence), and between $u_{\text {Bido }}$ and $u_{\text {Mono }}: \theta$ denotes the angle between the fibers direction and the $x$ axis.

\begin{tabular}{|c|c|c|c|c|c|c|c|}
\hline \multirow{2}{*}{\multicolumn{2}{|c|}{$\delta_{s, t}$}} & \multicolumn{3}{|c|}{$u^{*}=u_{\mathrm{DD}}$} & \multicolumn{3}{|c|}{$u^{*}=u_{\text {Mono }}$} \\
\hline & & $\Omega_{\mathrm{B}}$ & $\Omega_{\mathrm{M}}$ & $\Omega$ & $\Omega_{\mathrm{B}}$ & $\Omega_{\mathrm{M}}$ & $\Omega$ \\
\hline$\theta=0$ & $\begin{array}{c}2 \text { it. } \\
\varepsilon=1 \mathrm{e}-8\end{array}$ & $\begin{array}{l}2.82 \mathrm{e}-3 \\
2.86 \mathrm{e}-3\end{array}$ & $\begin{array}{l}2.44 \mathrm{e}-2 \\
2.44 \mathrm{e}-2\end{array}$ & $\begin{array}{l}1.74 \mathrm{e}-2 \\
1.73 \mathrm{e}-2\end{array}$ & $2.42 \mathrm{e}-2$ & $5.05 \mathrm{e}-2$ & $3.96 \mathrm{e}-2$ \\
\hline$\theta=\frac{\pi}{4}$ & $\begin{array}{c}2 \text { it. } \\
\varepsilon=1 \mathrm{e}-8\end{array}$ & $\begin{array}{l}1.87 \mathrm{e}-3 \\
1.68 \mathrm{e}-3\end{array}$ & $\begin{array}{l}4.36 \mathrm{e}-2 \\
4.42 \mathrm{e}-2\end{array}$ & $\begin{array}{l}3.09 \mathrm{e}-2 \\
3.13 \mathrm{e}-2\end{array}$ & $2.95 \mathrm{e}-2$ & $7.95 \mathrm{e}-2$ & $5.99 \mathrm{e}-2$ \\
\hline
\end{tabular}
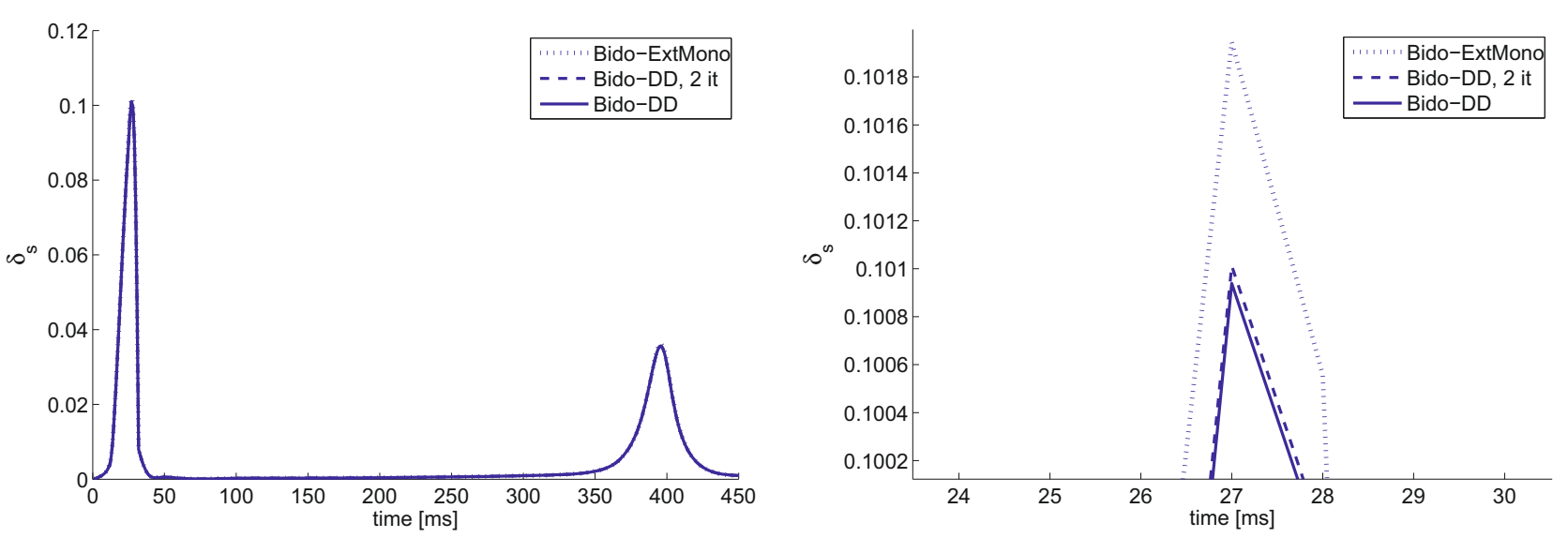

Figure 6. Left: relative differences in space between $u_{\text {Bido }}$ and $u_{\mathrm{EM}}$ (dotted line), and between $u_{\text {Bido }}$ and $u_{\mathrm{DD}}$, with two Schwarz iterations per time step (dashed line) and at convergence (solid line). Right: detail.

In order to have a global estimation of the error over the time interval of simulation we compute also the index

$$
\delta_{s, t}=\left(\frac{\int_{0}^{T}\left\|u_{\text {Bido }}-u^{*}\right\|_{L^{2}(V)}^{2} \mathrm{~d} t}{\int_{0}^{T}\left\|u_{\text {Bido }}-u^{\mathrm{rest}}\right\|_{L^{2}(V)}^{2} \mathrm{~d} t}\right)^{1 / 2},
$$

where $V$ is time to time $L^{2}\left(\Omega_{\mathrm{B}}\right), L^{2}\left(\Omega_{\mathrm{M}}\right)$, and $L^{2}(\Omega)$. In Table 2 we report the relative difference in space and time between $u_{\text {Bido }}$ and $u_{\mathrm{DD}}$, after two iterations of the Schwarz Algorithm and at convergence $\left(\| \boldsymbol{\eta}_{1}^{p+1}-\right.$ $\left.\eta_{1}^{p} \|_{l^{2}(\Gamma)}<\varepsilon\right)$, and between $u_{\text {Bido }}$ and $u_{\text {Mono }}$. The subdomains are $\Omega_{\mathrm{B}}=[0,1] \times[0,1]$ and $\Omega_{\mathrm{M}}=[1,2] \times[0,1]$. We report the relative differences computed over the domains $\Omega_{\mathrm{B}}, \Omega_{\mathrm{M}}$ and $\Omega$, for two different fibers orientations, one orthogonal and the other with an angle of $\theta=\pi / 4$ with respect to the interface. The time interval of the simulation is $[0,450] \mathrm{ms}$.

We can infer from Table 2 that the solution obtained from the Schwarz Algorithms proves to be more accurate than the pure Monodomain one both locally within $\Omega_{\mathrm{B}}$ and globally in $\Omega$. Moreover, the fibers orientation with respect to the interface does not affect significantly the results. On the other hand, Table 2 and Figure 6 show that there is neither a global improvement in space and time nor an appreciable difference along time between running the Schwarz Algorithm until convergence and running only two iterations of it.

Moreover, in Table 3 we report the relative CPU time with respect to a full Bidomain simulation: the $53 \%$ gain in the overall computational time is significant. Therefore, for the sake of computational cost reduction, 
TABLE 3. Relative CPU time with respect to a full Bidomain simulation. Here $u_{\mathrm{DD}}$ is computed with two Schwarz iterations.

\begin{tabular}{c|c|c|c}
\hline & $u_{\text {Bido }}$ & $u_{\text {DD }}$ & $u_{\text {Mono }}$ \\
\hline CPU Time & 1.00 & 0.47 & 0.15 \\
\hline
\end{tabular}

TABLE 4. Relative difference in space and time between $u_{\mathrm{EM}}$ and $u_{\mathrm{DD}}$ (at convergence), and between $u_{\mathrm{EM}}$ and $u_{\mathrm{Bido}}: \theta$ denotes the angle between the fibers direction and the $x$ axis.

\begin{tabular}{c|ccc|ccc}
\hline$\left\|u_{\mathrm{EM}}-u^{*}\right\|_{L^{2}(0, T ; V)}$ & \multicolumn{3}{|c|}{$u^{*}=u_{\mathrm{DD}}$} & \multicolumn{3}{c}{$u^{*}=u_{\text {Bido }}$} \\
\cline { 2 - 7 }$\left\|u_{\mathrm{EM}}-u^{\mathrm{rest}}\right\|_{L^{2}(0, T ; V)}$ & $\Omega_{\mathrm{B}}$ & $\Omega_{\mathrm{M}}$ & $\Omega$ & $\Omega_{\mathrm{B}}$ & $\Omega_{\mathrm{M}}$ & $\Omega$ \\
\hline$\theta=0$ & $5.31 \mathrm{e}-4$ & $1.21 \mathrm{e}-3$ & $9.34 \mathrm{e}-4$ & $2.46 \mathrm{e}-3$ & $2.47 \mathrm{e}-2$ & $1.75 \mathrm{e}-2$ \\
\hline$\theta=\frac{\pi}{4}$ & $2.70 \mathrm{e}-4$ & $1.96 \mathrm{e}-3$ & $1.40 \mathrm{e}-3$ & $1.48 \mathrm{e}-3$ & $4.49 \mathrm{e}-2$ & $3.17 \mathrm{e}-2$ \\
\hline
\end{tabular}

we will perform in practical applications (such as the one in Sect. 5.3) only two iterations of the Optimized Schwarz Algorithm.

\section{Comparison with the Extended Monodomain}

The second interface condition in (3.6) relies on approximating the unknown $u_{e, M}$. In order to highlight the error due to this approximation, we compare the domain decomposition solution $u_{\mathrm{DD}}$ with the solution $u_{\mathrm{EM}}$ obtained coupling the Bidomain and the Extended Monodomain through conditions (3.4) and (3.5). The corresponding Schwarz algorithm is implemented until convergence. We consider here again $\Omega_{\mathrm{B}}=[0,1] \times[0,1]$ and $\Omega_{\mathrm{M}}=[1,2] \times[0,1]$, and we run the simulation for $450 \mathrm{~ms}$. We plot in Figure 6 (right) the relative difference in space between $u_{\text {Bido }}$ and $u_{\mathrm{EM}}, u_{\text {Bido }}$ and $u_{\mathrm{DD}}$ (with both two Schwarz iterations and at convergence) as a function of time. The curves are nearly overlapped, showing analogous performance between the Bidomain/Monodomain coupling and the Bidomain/Extended Monodomain one. As a matter of fact (Fig. 6, right), very small difference between the two kinds of coupling are present.

Finally, we report in Table 4 the relative difference in space and time between $u_{\mathrm{EM}}$ and $u_{\mathrm{DD}}$ for two different fibers orientations, one orthogonal and the other with an angle of $\theta=\pi / 4$ with respect to the interface. In both cases $u_{\mathrm{DD}}$ is very close to $u_{\mathrm{EM}}$. Moreover, since $u_{\mathrm{EM}}$ computes also the extracellular potential, from Table 4 we can infer that the main error between $u_{\mathrm{DD}}$ and $u_{\text {Bido }}$ comes from the Monodomain approximation, and not from the extracellular potential approximation in the interface coupling.

\subsection{A three dimensional application}

In many applications the Monodomain model is accurate enough to capture the desired dynamics and effects of the action potential propagation. Potse et al. [29] stated that "in absence of applied currents, propagating of action potentials on the scale of a human heart can be studied with a monodomain model". Our idea is to solve the Bidomain equations only in those regions where the presence of applied currents cannot be treated with the Monodomain model. We simulate the propagation of the stimulus exerted by a bipolar pacemaker $[19,32]$ on a simplified left ventricular geometry, constituted by a truncated ellipsoid [7], with the analytical fibers mapping described therein. As usual we denote with $\Omega$ the computational domain, that we split in the two subdomains $\Omega_{\mathrm{B}}$ and $\Omega_{\mathrm{M}}$ (Fig. 7). We discretize the computational domain with a tetrahedral mesh, whose average element diameter is $0.6 \mathrm{~mm}$ : although this mesh size is not fine enough for an accurate capturing of the action potential propagation, it is sufficient to have a reasonable comparison between the solutions obtained with the different approaches considered.

We consider an epicardial pacemaker stimulation. In such situation the pacemaker lead reaches the left ventricle through a branch of the coronary sinus, thus its dipole is located on the exterior of the ventricle wall. This provides a stimulus through a variation of the extracellular current. The adoption of the Monodomain model in this case is not recommended since the Monodomain is unable to correctly capture the changes 


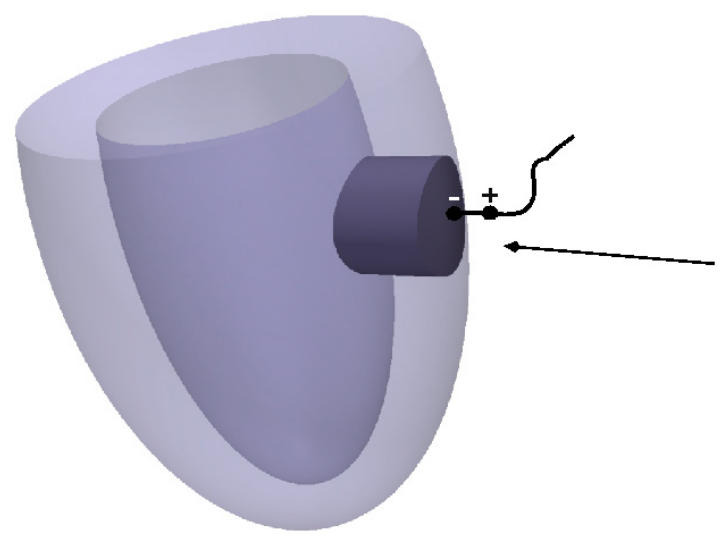

Figure 7 . The domains $\Omega_{\mathrm{B}}$ (dark) and $\Omega_{\mathrm{M}}$ (light), and the pacemaker lead. The arrow points out the visualization angle in the subsequent plots of the solution.

in the extracellular current [37]. This will be confirmed by our simulations. We consider two point sources, representing the cathode and the anode, located $5 \mathrm{~mm}$ apart in $\boldsymbol{x}_{c}=(2.75,0,0)$ and $\boldsymbol{x}_{a}=(3.25,0,0)$ respectively. The distance between the cathode and the epicardial wall is $0.5 \mathrm{~mm}$. We assume the electrodes to be immersed in an isotropic medium of conductance $g$. The electrical potential $\varphi$ satisfies the equation

$$
-\nabla \cdot(g \nabla \varphi)=I^{\mathrm{stim}}\left(\delta\left(\boldsymbol{x}-\boldsymbol{x}_{c}\right)-\delta\left(\boldsymbol{x}-\boldsymbol{x}_{a}\right)\right)
$$

where $\delta(\boldsymbol{x})$ is the Dirac distribution centred in $\mathbf{0}$ [19]. We take $I^{\text {stim }}=-4 \mathrm{~mA}$ for the first millisecond of the simulation, and 0 afterwards. Hence, solving the above equation in $\mathbb{R}^{3}$ and prescribing that $\varphi$ vanishes at infinity, we get

$$
\varphi=\frac{I^{\mathrm{stim}}}{4 \pi g}\left(\frac{1}{\left|\boldsymbol{x}-\boldsymbol{x}_{c}\right|}-\frac{1}{\left|\boldsymbol{x}-\boldsymbol{x}_{a}\right|}\right)
$$

We thus solve the Bidomain Problem (2.8), with boundary conditions

$$
\begin{aligned}
& \mathbf{n}^{T} \mathbf{D}_{i}\left(\nabla u_{B}+\nabla u_{e, B}\right)=0 \\
& \mathbf{n}^{T} \mathbf{D}_{e} \nabla u_{e, B}=\mathbf{n}^{T} g \nabla \varphi=-\frac{I^{\mathrm{stim}}}{4 \pi} \mathbf{n}^{T}\left(\frac{\boldsymbol{x}-\boldsymbol{x}_{c}}{\left|\boldsymbol{x}-\boldsymbol{x}_{c}\right|^{3}}-\frac{\boldsymbol{x}-\boldsymbol{x}_{a}}{\left|\boldsymbol{x}-\boldsymbol{x}_{a}\right|^{3}}\right)
\end{aligned}
$$

and no applied current $\left(I^{\text {app }}=\tilde{I}^{\text {app }}=0\right)$. Notice that $\int_{\partial \Omega} \mathbf{n}^{T} g \nabla \varphi \mathrm{d} \Sigma=\int_{\Omega} \nabla \cdot(g \nabla \varphi) \mathrm{d} \boldsymbol{x}=0$, since the point sources are outside the truncated ellipsoid (Gauss' law). This implies that the compatibility conditions on the second equation of the Bidomain problem is fulfilled. Again we ask $u_{e, B}$ to have zero mean.

We take the Bidomain region $\Omega_{\mathrm{B}}$ close to the electrodes. More precisely, $\Omega_{\mathrm{B}}$ is given by the intersection of a cylinder of radius $r=0.75 \mathrm{~cm}$ and axis $x$ with the truncated ellipsoid, as shown in Figure 7. On $\partial \Omega_{\mathrm{B}} \cap \partial \Omega$ we prescribe the boundary conditions (5.3). We couple the two domains with the Schwarz algorithm proposed, performing only two iterations per time step. The parameter $\alpha^{*}$ is the one given in (4.26). If we face this problem with the Monodomain equation over the entire domain, by the usual assumption $\mathbf{D}_{e}=\frac{\sigma_{e}^{l}}{\sigma_{i}^{l}} \mathbf{D}_{i}$ the boundary conditions (5.3) read

$$
\begin{aligned}
\mathbf{n}^{T} \mathbf{D}_{M} \nabla u & =\mathbf{n}^{T} \frac{\sigma_{e}^{l}}{\sigma_{i}^{l}+\sigma_{e}^{l}} \mathbf{D}_{i}\left(\nabla u_{B}+\nabla u_{e, B}\right)-\mathbf{n}^{T} \frac{\sigma_{i}^{l}}{\sigma_{i}^{l}+\sigma_{e}^{l}} \mathbf{D}_{e} \nabla u_{e, B} \\
& =-\mathbf{n}^{T} \frac{g \sigma_{i}^{l}}{\sigma_{i}^{l}+\sigma_{e}^{l}} \nabla \varphi=\frac{I^{\mathrm{stim}} \sigma_{i}^{l}}{4 \pi\left(\sigma_{i}^{l}+\sigma_{e}^{l}\right)} \mathbf{n}^{T}\left(\frac{\boldsymbol{x}-\boldsymbol{x}_{c}}{\left|\boldsymbol{x}-\boldsymbol{x}_{c}\right|^{3}}-\frac{\boldsymbol{x}-\boldsymbol{x}_{a}}{\left|\boldsymbol{x}-\boldsymbol{x}_{a}\right|^{3}}\right) .
\end{aligned}
$$




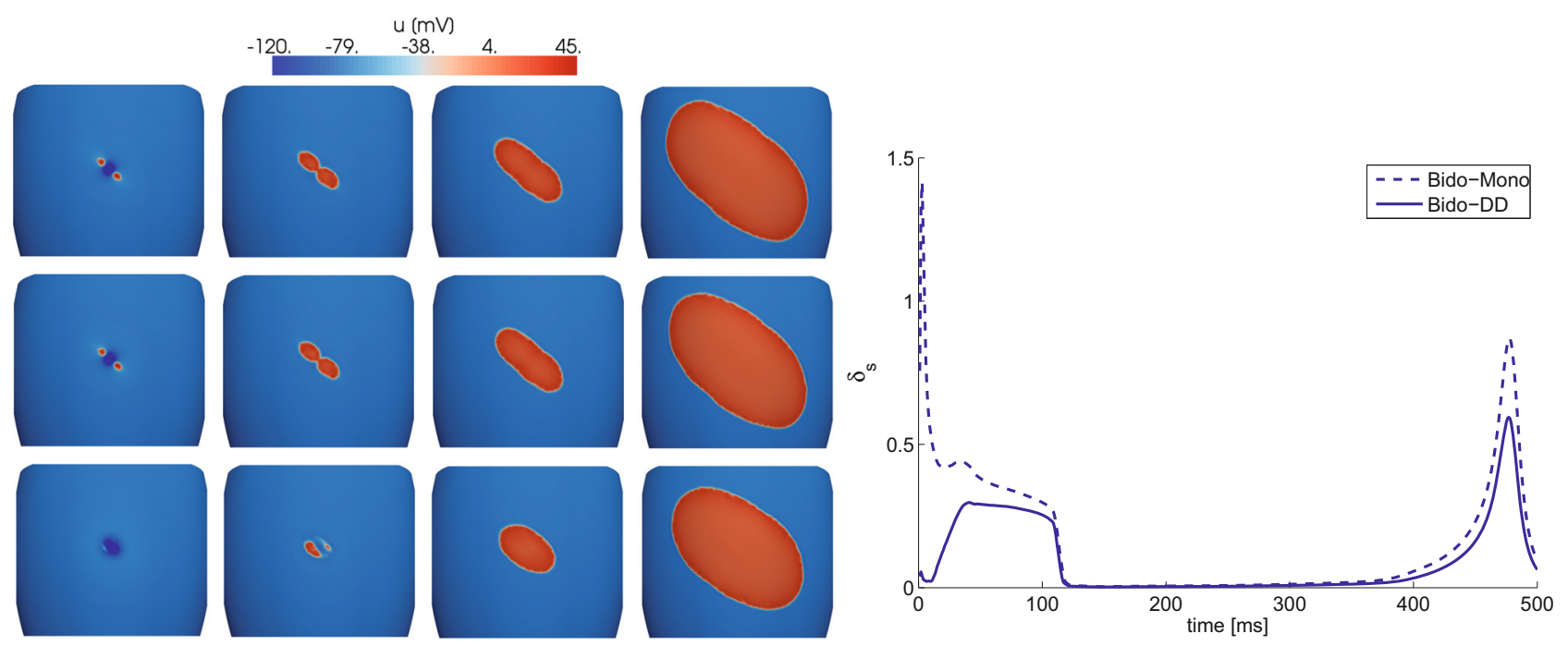

FiguRE 8. Left: transmembrane potential $[\mathrm{mV}]$ (top to bottom: $u_{\text {Bido }}, u_{\mathrm{DD}}, u_{\mathrm{Mono}}$ ) in the area enlightened by the arrow in Figure 7 at different time-steps (from left to right: $t=2 \mathrm{~ms}$, $t=5 \mathrm{~ms}, t=10 \mathrm{~ms}, t=25 \mathrm{~ms}$ ). Right: relative differences in space (on $\Omega$ ), as a function of time, between $u_{\text {Bido }}$ and $u_{\text {Mono }}$ (dashed line) and between $u_{\text {Bido }}$ and $u_{\mathrm{DD}}$ (solid line).
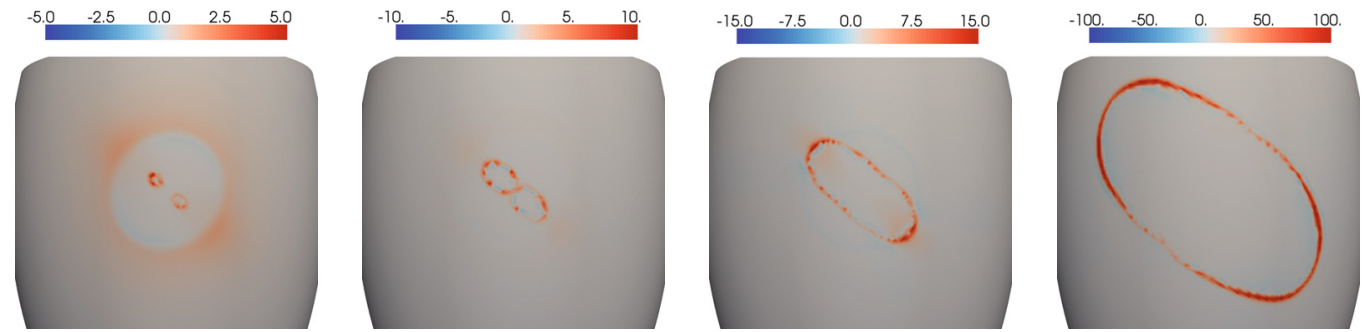

FigurE 9. Difference between $u_{\text {Bido }}$ and $u_{\mathrm{DD}}[\mathrm{mV}]$ in the area enlightened by the arrow in Figure 7 at different time-steps (from left to right: $t=2 \mathrm{~ms}, t=5 \mathrm{~ms}, t=10 \mathrm{~ms}, t=25 \mathrm{~ms}$ ). $u_{\mathrm{DD}}$ is computed with two Schwarz iterations.

In Figure 8 (left) we report the transmembrane potential on the epicardial wall near the stimulus region (the area enlightened by the arrow in Fig. 7) computed with different models. In Figure 8 (right) we plot the relative difference in space, as a function of time, between $u_{\text {Bido }}$ and $u_{\text {Mono }}$ and $u_{\text {Bido }}$ and $u_{\mathrm{DD}}$. When the depolarization and repolarization waves travel in $\Omega_{\mathrm{B}}$, the error $u_{\mathrm{DD}}$ is of the order of $50 \%$ of $u_{\mathrm{Bido}}$. This is much larger than that in the $2 \mathrm{D}$ case, since now $\left|\Omega_{\mathrm{B}}\right| \gg\left|\Omega_{\mathrm{B}}\right|$. In Figure 9 we report the difference between $u_{\mathrm{Bido}}$ and $u_{\mathrm{DD}}$.

Table 5 shows that both $u_{\mathrm{DD}}$ and $u_{\mathrm{EM}}$ are more accurate solutions than $u_{\mathrm{Mono}}$, especially within $\Omega_{\mathrm{B}}$. The solutions $u_{\mathrm{DD}}$ and $u_{\mathrm{EM}}$ are comparable in terms of accuracy, but the latter one is computationally more expensive (Tab. 6); it is actually more expensive than the Bidomain solution itself. This is due to the fact that to get $u_{\mathrm{EM}}$ one has to solve the ill-conditioned system (2.16).

The computational costs associated with the Schwarz algorithm and the full Monodomain approach are similar and significantly smaller than the full Bidomain one. Thus if one wants to properly solve the coupling between the myocardium and the bipolar pacemaker, the Schwarz algorithm is a cheap alternative to the full 
TABLE 5 . Relative difference in space and time between $u_{\text {Bido }}$ and $u_{\mathrm{DD}}, u_{\text {Bido }}$ and $u_{\text {Mono }}, u_{\text {Bido }}$ and $u_{\mathrm{EM}}$.

\begin{tabular}{c|ccc|ccc|ccc}
\hline & \multicolumn{3}{|c|}{$u^{*}=u_{\mathrm{DD}}$} & \multicolumn{3}{c|}{$u^{*}=u_{\mathrm{EM}}$} & \multicolumn{3}{c}{$u^{*}=u_{\mathrm{Mono}}$} \\
\cline { 2 - 9 } & $\Omega_{\mathrm{B}}$ & $\Omega_{\mathrm{M}}$ & $\Omega$ & $\Omega_{\mathrm{B}}$ & $\Omega_{\mathrm{M}}$ & $\Omega$ & $\Omega_{\mathrm{B}}$ & $\Omega_{\mathrm{M}}$ & $\Omega$ \\
\hline$\delta_{s, t}$ & $8.67 \mathrm{e}-3$ & $1.14 \mathrm{e}-1$ & $1.13 \mathrm{e}-1$ & $7.34 \mathrm{e}-3$ & $1.14 \mathrm{e}-1$ & $1.13 \mathrm{e}-1$ & $5.41 \mathrm{e}-2$ & $1.42 \mathrm{e}-1$ & $1.40 \mathrm{e}-1$ \\
\hline
\end{tabular}

TABLE 6. Relative CPU time. $u_{\mathrm{DD}}$ and $u_{\mathrm{EM}}$ computed with two Schwarz iterations.

\begin{tabular}{c|c|c|c|c}
\hline & $u_{\text {Bido }}$ & $u_{\text {DD }}$ & $u_{\text {EM }}$ & $u_{\text {Mono }}$ \\
\hline CPU Time & 1.000 & 0.309 & 1.400 & 0.308 \\
\hline
\end{tabular}

Bidomain approach. In this case, the full Monodomain approach is infeasible since the Monodomain equation fails to handle the extracellular potential.

\section{Conclusions}

Reduction of computational costs from solving the Bidomain equations in electrocardiology is usually attained by means of parallel hardware architectures. In this paper we address a different (complementary rather than alternative) approach essentially based on downscaling the expensive Bidomain model to the simpler Monodomain one on (one or more) parts of the computational domain supposed to be less relevant to the entire potential dynamics. Coupling the Bidomain system with the Monodomain equation is by itself a nontrivial task, since the two problems have different mathematical features. In particular, the Monodomain problem solves the transmembrane potential, while the Bidomain one considers the intra- and extracellular potentials separately. In this paper we presented some possible interface conditions obtained started from a Bidomain problem solved by subdomains. Moving from classical interface conditions, we downscaled them in a natural way as a consequence of the approximation with the Monodomain problem. Robin-Robin conditions have been used in the context of an Optimized Schwarz Algorithm. Fourier analysis allowed to prove the convergence and to find a good approximation of the optimal values for the parameter in the interface condition. Numerical results carried out in both $2 \mathrm{D}$ and $3 \mathrm{D}$ confirmed that our DD approach is a viable method for reducing CPU costs without affecting significantly the accuracy. Due to the fast convergence of the Schwarz Algorithm, the DD approach is effective whenever we are interested in solving the Bidomain model only on specific regions of the domain, as in the presence of pacemakers. Another promising application is the development of a model adaptive solver. Dynamics of the differences between the DD and the fully Bidomain system suggest to investigate a posteriori error estimates in view of an adaptive implementation of the algorithms presented here. An effective and reliable a posteriori error estimator has been presented in [23], where the downscaled model was the Extended Monodomain. However, our numerical results showed that the solution computed with the Bidomain/Monodomain DD approach is pretty close to that computed with the Bidomain/Extended Monodomain DD approach. The implementation of the Optimized Schwarz Algorithm presented here in a model adaptive strategy to solve real problems with mesh sizes fine enough to capture the details of the action potential propagation are an ongoing development of the present work and will be subject of a forthcoming paper.

Acknowledgements. The authors wish to thank Lucia Mirabella (Politecnico di Milano and Emory University) for many fruitful discussions in preparing this work. 


\section{REFERENCES}

[1] A. Alonso-Rodriguez and L. Gerardo-Giorda, New non-overlapping domain decomposition methods for the time-harmonic Maxwell system. SIAM J. Sci. Comp. 28 (2006) 102-122.

[2] M. Bendahmane and K.H. Karlsen, Analysis of a class of degenerate reaction-diffusion systems and the bidomain model of cardiac tissue. Netw. Heterog. Media 1 (2006) 185-218.

[3] Y. Bourgault, Y. Coudière and C. Pierre, Existence and uniqueness of the solution for the bidomain model used in cardiac electrophysiology. Nonlinear Anal.: Real World Appl. 10 (2009) 458-482.

[4] R.H. Clayton and A.V. Panfilov, A guide to modelling cardiac electrical activity in anatomically detailed ventricles. Prog. Biophys. Mol. Biol. 96 (2008) 19-43.

[5] R.H. Clayton, O.M. Bernus, E.M. Cherry, H. Dierckx, F.H. Fenton, L. Mirabella, A.V. Panfilov, F.B. Sachse, G. Seemann and H. Zhang, Models of cardiac tissue electrophysiology: Progress, challenges and open questions. Prog. Biophys. Mol. Biol. (2010) DOI: 10.1016/j.pbiomolbio.2010.05.008.

[6] J.C. Clements, J. Nenonen, P.K.J. Li and M. Horacek, Activation dynamics in anisotropic cardiac tissue via decoupling. Ann. Biomed. Eng. 32 (2004) 984-990.

[7] P. Colli Franzone and L.F. Pavarino, A parallel solver for reaction-diffusion systems in computational electrocardiology. Math. Mod. Meth. Appl. Sci. 14 (2004) 883-911.

[8] P. Colli Franzone and G. Savaré, Degenerate evolution systems modeling the cardiac electric field at micro and macroscopic level, in Evolution Equations, Semigroups and Functional Analysis, A. Lorenzi and B. Ruf Eds., Birkhauser (2002) 49-78.

[9] P. Colli Franzone, L.F. Pavarino and B. Taccardi, Simulating patterns of excitation, repolarization and action potential duration with cardiac Bidomain and Monodomain models. Math. Biosc. 197 (2005) 35-66.

[10] P. Colli Franzone, P. Deuflhard, B. Erdmann, J. Lang and L.F. Pavarino, Adaptivity in space and time for reaction-diffusion systems in electrocardiology. SIAM J. Sci. Comput. 28 (2006) 942-962.

[11] V. Dolean and F. Nataf, An Optimized Schwarz Algorithm for the compressible Euler equations, in Domain Decomposition Methods in Science and Engineering, Proceedings of the DD16 Conference, Springer-Verlag (2007) 173-180.

[12] V. Dolean, M.J. Gander and L. Gerardo-Giorda, Optimized Schwarz Methods for Maxwell's equations. SIAM J. Sci. Comput. 31 (2009) 2193-2213.

[13] J.J. Fox, J.L. McHarg and R.F. Gilmour, Ionic mechanism of electrical alternans. Am. J. Physiol. (Heart Circ. Physiol.) 282 (2002) H516-H530.

[14] M.J. Gander, Optimized Schwarz methods. SIAM J. Num. Anal. 44 (2006) 699-731.

[15] M.J. Gander, F. Magoulès and F. Nataf, Optimized Schwarz methods without overlap for the Helmholtz equation. SIAM J. Sci. Comput. 24 (2002) 38-60.

[16] L. Gerardo-Giorda, L. Mirabella, F. Nobile, M. Perego and A. Veneziani, A model-based block-triangular preconditioner for the Bidomain system in electrocardiology. J. Comp. Phys. 228 (2009) 3625-3639.

[17] J.P. Keener, Direct activation and defibrillation of cardiac tissue. J. Theor. Biol. 178 (1996) 313-324.

[18] J.P. Keener and J. Sneyd, Mathematical Physiology. Springer-Verlag, New York (1998).

[19] D.C. Latimer and B.J. Roth, Electrical stimulation of cardiac tissue by a bipolar electrode in a conductive bath. IEEE Trans. Biomed. Eng. 45 (1998) 1449-1458.

[20] J. Le Grice, B.H. Smaill, L.Z. Chai, S.G. Edgar, J.B. Gavin and P.J. Hunter, Laminar structure of the heart: ventricular myocyte arrangement and connective tissue architecture in the dog. Am. J. Physiol. (Heart Circ. Physiol.) 269 (1995) H571H582.

[21] P.-L. Lions, On the Schwarz alternating method. III: A variant for nonoverlapping subdomains, in Third International Symposium on Domain Decomposition Methods for Partial Differential Equations, held in Houston, Texas, March 20-22, 1989, Philadelphia, R. Glowinski, J. Périaux, T.F. Chan and O. Widlund Eds., SIAM (1990).

[22] L. Luo and Y. Rudy, A model of the ventricular cardiac action potential: depolarization, repolarization and their interaction. Circ. Res. 68 (1991) 1501-1526.

[23] L. Mirabella, F. Nobile and A. Veneziani, An a posteriori error estimator for model adaptivity in electrocardiology. Technical Report TR-2009-025, Dept. MathCS, Emory University (2009).

[24] B.F. Nielsen, T.S. Ruud, G.T. Lines and A. Tveito, Optimal monodomain approximation of the bidomain equations. Appl. Math. Comp. 184 (2007) 276-290.

[25] A. Nygren, C. Fiset, L. Firek, J.W. Clark, D.S. Lindblad, R.B. Clark and W.R. Giles, Mathematical model of an adult human atrial cell: the role of $K^{+}$currents in repolarization. Circ. Res. 82 (1998) 63-81.

[26] L.F. Pavarino and S. Scacchi, Multilevel additive Schwarz preconditioners for the Bidomain reaction-diffusion system. SIAM J. Sci. Comp. 31 (2008) 420-443.

[27] M. Pennacchio and V. Simoncini, Efficient algebraic solution of rection-diffusion systems for the cardiac excitation process. J. Comput. Appl. Math. 145 (2002) 49-70.

[28] M. Perego and A. Veneziani, An efficient generalization of the Rush-Larsen method for solving electro-physiology membrane equations. Electronic Transaction on Numerical Analysis 35 (2009) 234-256. 
[29] M. Potse, B. Dubé, J. Richer and A. Vinet, A comparison of monodomain and bidomain reaction-diffusion models for action potential propagation in the human heart. IEEE Trans. Biomed. Eng. 53 (2006) 2425-2435.

[30] A. Quarteroni and A. Valli, Domain Decompostion Methods for Partial Differential Equations. Oxford University Press, Oxford (1999).

[31] A. Quarteroni, L. Formaggia and A. Veneziani, Complex Systems in Biomedicine, in Computational electrocardiology: mathematical and numerical modeling, P. Colli Franzone, L. Pavarino and G. Savaré Eds., Springer, Milan (2006).

[32] B. Roth, A comparison of two boundary conditions used with the bidomain model of cardiac tissue. Ann. Biomed. Eng. 19 (1991) 669-678.

[33] S. Scacchi, A hybrid multilevel Schwarz method for the bidomain model. Comp. Meth. Appl. Mech. Eng. 197 (2008) $4051-4061$.

[34] B.F. Smith, P.E. Bjørstad and W. Gropp, Domain Decomposition: Parallel Multilevel Methods for Elliptic Partial Differential Equations. Cambridge University Press, Cambridge (1996).

[35] D. Streeter, Gross morphology and fiber geometry in the heart, in Handbook of Physiology 1 (Sect. 2), R.M. Berne Ed., Williams and Wilnkins (1979) 61-112.

[36] A. Toselli and O. Widlund, Domain Decomposition Methods. 1st edition, Springer (2004).

[37] N. Trayanova, Defibrillation of the heart: insights into mechanisms from modelling studies. Exp. Physiol. 91 (2006) $323-337$.

[38] M. Veneroni, Reaction-diffusion systems for the macroscopic bidomain model of the cardiac electric field. Nonlinear Anal.: Real World Appl. 10 (2009) 849-868.

[39] E.J. Vigmond, F. Aguel and N.A. Trayanova, Computational techniques for solving the bidomain equations in three dimensions. IEEE Trans. Biomed. Eng. 49 (2002) 1260-1269.

[40] E.J. Vigmond, R. Weber dos Santos, A.J. Prassl, M. Deo and G. Plank, Solvers for the caridac bidomain equations. Prog. Biophys. Mol. Biol. 96 (2008) 3-18.

[41] R. Weber dos Santos, G. Planck, S. Bauer and E.J. Vigmond, Parallel multigrid preconditioner for the cardiac bidomain model. IEEE Trans. Biomed. Eng. 51 (2004) 1960-1968. 